\title{
A Structure-Preserving Pivotal Method for Affine Variational Inequalities
}

\author{
Youngdae Kim * $\quad$ Olivier Huber * Michael C. Ferris *
}

October 8, 2018

\begin{abstract}
Affine variational inequalities (AVI) are an important problem class that generalize systems of linear equations, linear complementarity problems and optimality conditions for quadratic programs. This paper describes PATHAVI, a structure-preserving pivotal approach, that can process (solve or determine infeasible) large-scale sparse instances of the problem efficiently, with theoretical guarantees and at high accuracy. PATHAVI implements a strategy that is known to process models with good theoretical properties without reducing the problem to specialized forms, since such reductions may destroy structure in the models and can lead to very long computational times. We demonstrate formally that PATHAVI implicitly follows the theoretically sound iteration paths, and can be implemented in a large scale setting using existing sparse linear algebra and linear programming techniques without employing a reduction. We also extend the class of problems that PATHAVI can process. The paper demonstrates the effectiveness of our approach by comparison to the PATH solver used on a complementarity reformulation of the AVI in the context of applications in friction contact and Nash Equilibria problems. PATHAVI is a general purpose solver, and freely available under the same conditions as PATH.
\end{abstract}

\footnotetext{
${ }^{*}$ Wisconsin Institute for Discovery and Department of Computer Sciences, University of Wisconsin-Madison, 1210 West Dayton St., Madison, WI, 53706

Y. Kim

Email: youngdae@cs.wisc.edu

O. Huber

Email: ohuber2@wisc.edu

M. C. Ferris

Email: ferris@cs.wisc.edu
} 


\section{Introduction}

In this paper, we present PATHAVI, a structure-preserving pivotal method for affine variational inequalities (AVIs) in $\mathbb{R}^{n}$. An $\operatorname{AVI}(C, q, M)$ is defined as follows: given a polyhedral convex set $C$, find $z \in C$ such that

$$
\langle M z+q, y-z\rangle \geq 0, \quad \forall y \in C
$$

where $M \in \mathbb{R}^{n \times n}, q \in \mathbb{R}^{n}$ and $\langle\cdot, \cdot\rangle$ is the usual Euclidean inner product. An AVI is a linear generalized equations [23] and we refer to [14] for results on existence, uniqueness, and stability theory for such systems.

PATHAVI tries to solve an $\operatorname{AVI}(C, q, M)$ by computing a zero of the normal map [24] associated with the AVI. The normal map $M_{C}: \mathbb{R}^{n} \rightarrow \mathbb{R}^{n}$ is defined as follows:

$$
M_{C}(x):=M\left(\pi_{C}(x)\right)+q+x-\pi_{C}(x)
$$

with $\pi_{C}(\cdot)$ denoting the Euclidean projector onto the set $C$. One can easily see that $M_{C}\left(x^{*}\right)=0$ if and only if $z^{*}=\pi_{C}\left(x^{*}\right)$ with $x^{*}=z^{*}-\left(M z^{*}+q\right)$ is a solution to the $\operatorname{AVI}(C, q, M)$. To compute a zero of $M_{C}(x)$, our method employs the complementary pivoting method [12, 19] with a ray start: the piecewise-linear (PL) map $G_{C}: \mathbb{R}^{n} \times \mathbb{R}_{+} \rightarrow \mathbb{R}^{n}$ is defined as

$$
G_{C}(x, t):=M_{C}(x)-t r
$$

with $r \in \mathbb{R}^{n}$ denoting the covering vector and $t$ the auxiliary variable. A path defined as $G_{C}^{-1}(0)$ is followed through complementary pivoting. The algorithm terminates when either $t$ becomes zero (a solution to the AVI is found) or a secondary ray is generated. Under some additional assumptions this latter outcome can be interpreted in terms of feasibility of the AVI.

This approach has been previously investigated in [6]. However the method in [6] requires a reduction transforming the given $\operatorname{AVI}(C, q, M)$ to a reduced $\operatorname{AVI}(\tilde{C}, \tilde{q}, \tilde{M})$ to eliminate lines in $C$ and solves the reduced AVI. The matrix $\tilde{M}$ is constructed from a Schur complement computation and the polyhedral constraints defining $\tilde{C}$ are computed by multiplying with orthonormal matrices. Thus the original structure in $C$ and $M$ may be lost: in particular if the AVI is sparse, there is no guarantee that the resulting reduced AVI would enjoy the same property. We provide an instance where this happens in Section 6.2. In sharp contrast, PATHAVI does not require any reduction at all. Therefore our method is able to take advantage of a sparse structure, whereas the method in [6] often needs to perform dense linear algebra computations.

The main challenge in tackling the problem in its original space lies in the starting phase. For good theoretical properties, a ray start is required, and it is well-defined at an extreme point. However, when $C$ contains lines there is no extreme point. To perform a ray start in that case, we need to find an implicit extreme point, which generalizes the notion of an extreme point when the underlying feasible region contains lines. Roughly speaking, if we project an implicit extreme point of $C$ on the subset where all lines are removed, we find an extreme point. We show that there is an implicit extreme point satisfying the sufficient conditions for a ray start. We explain how the phase 1 of the simplex method can be used to find such a point.

Regarding processability, we show that PATHAVI can process an $\operatorname{AVI}(C, q, M)$ whenever $M$ is an $L$ matrix with respect to the recession cone of $C$ [6, Definition 4.2]. We also exhibit two new classes of AVI where PATHAVI finds a solution. The first one stems from the study of friction contact problems from an AVI perspective, and the second one can be seen as a generalization of a known existence result for LCP for copositive matrices. In contrast with the previous results, the conditions are on both $M$ and $q$.

One of the practical and most widely used method for solving an AVI has been to use the PATH solver [8], which is one of the most robust and efficient solvers for mixed complementarity problems (MCPs). It is well 
known [10, 14] that an AVI can be reformulated as a linear MCP, and PATH uses this approach when it solves an AVI. However the MCP reformulation does not exploit the polyhedral structure of the set $C$, in that complementary pivoting of PATH is done over a different PL-manifold from PATHAVI's. We compare theoretical properties of the two formulations, and present computational results comparing performance of the solvers.

This paper is organized as follows: in Section 2, we briefly describe how one uses the complementary pivoting method on a PL-manifold to compute a zero of the normal map associated with a given AVI. Section 3 presents our main theoretical results: firstly, we discuss sufficient conditions for a ray start, we define an implicit extreme point, and prove the existence of an implicit extreme point satisfying the conditions for a ray start. Secondly, we show that PATHAVI can process $L$-matrices and we show new types of AVIs processable by PATHAVI. In Section 4 , we present the computational procedure to start PATHAVI. Section 5 introduces the MCP reformulation of the AVI and analyzes worst-case performance of the two formulations. Finally we present computational results in Section 6, and Section 7 concludes this paper.

A word about our notation is in order. Let $S$ be a convex set in $\mathbb{R}^{n}$. The lineality space of $S$ is denoted by $\operatorname{lin} S$. The symbol ri $S$ denotes the relative interior of $S$. The affine hull of $S$ is denoted by aff $S$. By par $S$, we mean the subspace parallel to aff $S$ such that aff $S=s+\operatorname{par} S$ for each $s \in S$. When ordered index sets are used as subscripts on a matrix, they define a submatrix: for ordered index sets $\alpha \subset\{1, \ldots, m\}$ and $\beta \subset\{1, \ldots, n\} M_{\alpha \beta}$ denotes a submatrix of $M$ consisting of rows and columns of $M$ in the order of $\alpha$ and $\beta$, respectively. When matrices are used as subscripts on a matrix, they define another matrix: for matrices $Q$ and $\bar{Q}$ having appropriate dimensions $M_{Q \bar{Q}}$ denotes $Q^{T} M \bar{Q}$. For an $\operatorname{AVI}(C, q, M)$, the set $C$ is assumed to be the set $\left\{z \in \mathbb{R}^{n} \mid A z-b \in K, l \leq z \leq u\right\}$ with $l_{j}, u_{j} \in \mathbb{R} \cup\{-\infty, \infty\}, b_{i} \in \mathbb{R}$ and $A_{i \bullet} \neq 0$ for $i=1, \ldots, m$ and $j=1, \ldots, n$, where the set $K$ is a Cartesian product of $\mathbb{R}_{+},\{0\}$, or $\mathbb{R}_{-}$to accommodate constraints of the form $\geq,=$, or $\leq$, respectively. For a closed convex cone $K$, the dual cone of $K$ is denoted by $K^{D}:=\{y \mid\langle y, k\rangle \geq 0, \forall k \in K\}$. For the rest of this paper, $Q$ and $\bar{Q}$ denote orthonormal basis matrices for the lineality space of $C$ and its orthogonal complement, respectively.

\section{Background}

In this section, we briefly describe how to compute a zero of the normal map associated with a given $\operatorname{AVI}(C, q, M)$ using the complementary pivoting method with a ray start. We also introduce some concepts related to processability of AVIs. Refer to [6, 12, 19, 24] for more details.

The basic procedure of the complementary pivoting method to compute a zero of the normal map associated with an $\operatorname{AVI}(C, q, M)$ is as follows: i) compute an initial solution $\left(x^{0}, t^{0}\right)$ such that $G_{C}\left(x^{0}, t^{0}\right)=0$, and the point $\left(x^{0}, t^{0}\right)$ lies on a ray, called a starting ray, consisting of points $(x(t), t)$ with $G_{C}(x(t), t)=0$ and $\pi_{C}(x(t))=\pi_{C}\left(x^{0}\right)$ for all $t \geq t^{0}$; then ii) starting from $\left(x^{0}, t^{0}\right)$ follow a path $G^{-1}(0)=\left\{(x, t) \in \mathbb{R}^{n} \times \mathbb{R}_{+} \mid\right.$ $G(x, t)=0\}$ using the complementary pivoting method until $t$ becomes zero or a secondary ray is generated. As we will see, PATHAVI generates a starting ray at an implicit extreme point of $C$, i.e., $\pi_{C}\left(x^{0}\right)$ is an implicit extreme point.

Computationally, finding an initial solution $\left(x^{0}, t^{0}\right)$ amounts to computing a complementary basic solution having $z=\pi_{C}\left(x^{0}\right)$ for the following system of equations:

$$
\begin{aligned}
M z+q-A^{T} \lambda-w+v & =0, \\
A z-b & =s,
\end{aligned}
$$


with complementarity between variables

$$
\begin{aligned}
K \ni s & \perp \quad \lambda \in K^{D}, \\
0 \leq z-l & \perp \quad w \geq 0, \\
0 \leq u-z & \perp \quad v \geq 0 .
\end{aligned}
$$

The complementary basic solution satisfies the sufficient conditions for a ray start as defined in Section 3 Then by adding $-t r$ with $r \in \operatorname{ri}\left(N_{C}\left(\pi_{C}\left(x^{0}\right)\right)\right)$ to the first equation in (2) and pivoting in the $t$ variable, we generate an almost complementary feasible basis and start complementary pivoting.

Geometrically, the map $G_{C}(x, t)$ is defined over a $\operatorname{PL}(n+1)$-manifold $\mathscr{M}_{C}$, where definition of a manifold follows from [12, Section 4]. The manifold $\mathscr{M}_{C}$ consists of a pair $\left(\mathbb{R}^{n} \times \mathbb{R}_{+},\left\{\sigma_{i} \times \mathbb{R}_{+} \mid i \in \mathscr{I}\right\}\right)$ such that each $\sigma_{i}$ is a set formed by $\sigma_{i}=F_{i}+N_{F_{i}}$, where $F_{i}$ is from a collection of the nonempty faces $\left\{F_{i} \mid i \in \mathscr{I}\right\}$ of $C$, and $N_{F_{i}}$ is a normal cone having constant value on ri $F_{i}$. The manifold $\mathscr{M}_{C}$ is constructed from the normal manifold $\mathscr{N}_{C}$ consisting of a pair $\left(\mathbb{R}^{n},\left\{\sigma_{i} \mid i \in \mathscr{I}\right\}\right)$ by doing a Cartesian product each $\sigma_{i}$ with $\mathbb{R}_{+}$. Note that the collection of the sets $\left\{\sigma_{i} \mid i \in \mathscr{I}\right\}$ is a subdivision of $\mathbb{R}^{n}$. Consequently, $\left\{\sigma_{i} \times \mathbb{R}_{+} \mid i \in \mathscr{I}\right\}$ is a subdivision of $\mathbb{R}^{n} \times \mathbb{R}_{+}$. The $k$-dimensional faces of the $\sigma_{i} \times \mathbb{R}_{+}$are called the $k$-cells of $\mathscr{M}_{C}$. Similarly, the $k$-dimensional faces of the $\sigma_{i}$ are called the $k$-cells of $\mathscr{N}_{C}$. The map $G_{C}$ coincides with some affine transformation on each $(n+1)$-cell $\sigma_{i} \times \mathbb{R}_{+}$as the normal map $M_{C}$ does on each $n$-cell $\sigma_{i}$ [24, Proposition 2.5]. Note that the starting ray $(x(t), t)$ for $t \geq t^{0}$ lies interior to some $(n+1)$-cell $\sigma_{i} \times \mathbb{R}_{+}$of $\mathscr{M}_{C}$, where $x^{0}$ is a regular point, i.e., $\operatorname{dim}\left(G_{C}\left(\sigma_{i} \times \mathbb{R}_{+}\right)\right)=n$. Under lexicographic pivoting, each complementary pivoting generates each piece of the 1 -manifold $G^{-1}(0)$ such that it starts from a boundary of a $(n+1)$-cell of $\mathscr{M}_{C}$ (except for the first piece containing the starting ray) and passes through interior to that cell until it reaches the cell's another boundary. If it does not reach a boundary, then we say that a secondary ray is generated. The set of $(n+1)$-cells the 1-manifold passes through never repeats. As there is a finite number of $(n+1)$ cells of $\mathscr{M}_{C}$, we have either $t$ reaches zero (equivalently we find a solution to the $\operatorname{AVI}(C, q, M)$ ) or a secondary ray is generated.

Processability is tied to the conditions under which a secondary ray occurs. As with the LCPs, the answer to this question involves specific matrix classes that we now define.

Definition 2.1 (Definition 4.1 [6]). Let $K$ be a closed convex cone. A matrix $M$ is said to be copositive with respect to $K$ if $\langle x, M x\rangle \geq 0$ for all $x \in K$. If furthermore it holds that for all $x \in K\langle x, M x\rangle=$ implies $\left(M+M^{T}\right) x=0$, then $M$ is copositive-plus.

Definition 2.2. Let $K$ be a closed convex cone. A matrix $M$ is said to be semimonotone with respect to $K$ if for every $q \in \operatorname{ri}\left(K^{D}\right)$, the solution set of the generalized complementarity problem

$$
z \in K, \quad M z+q \in K^{D}, \quad z^{T}(M z+q)=0
$$

is contained in lin $K$.

Remark. This definition is consistent with the existing semimonotone property in the LCP, as given in 7 Definition 3.9.1]. In this case $K=\mathbb{R}_{+}^{n}$ and $\operatorname{lin} K=\{0\}$. Then the condition (3) is equivalent to 0 being the solution set of $\operatorname{LCP}(M, q)$ for all $q>0$, which by Theorem 3.9.3 in [7] is equivalent to the standard definition of $M$ semimonotone.

Definition 2.3 (Definition $4.2[6]$ ). Let $K$ be a closed convex cone. A matrix $M$ is said to be an L-matrix with respect to $K$ if both

(a) $M$ is semimonotone with respect to $K$ 
(b) For any $z \neq 0$ satisfying

$$
z \in K, \quad M z \in K^{D}, \quad z^{T} M z=0,
$$

there exists $z^{\prime} \neq 0$ such that $z^{\prime}$ is contained in every face of $K$ containing $z$ and $-M^{T} z^{\prime}$ is contained in every face of $K^{D}$ containing $M z$.

Lemma 2.1 (Lemma 4.5 [6]). If a matrix $M$ is copositive-plus with respect to a closed convex cone $K$, then it is an L-matrix with respect to $K$.

The main existing result on the processability using a pivotal method is the following.

Theorem 2.1 (Theorem 4.4 [6]). Suppose that $C$ is a polyhedral convex set, and $M$ is an L-matrix with respect to rec $C$ which is invertible on the lineality space of $C$. Then exactly one of the following occurs:

- The method of [6] solves the AVI $(C, q, M)$.

- The following system has no solution

$$
M z+q \in(\operatorname{rec} C)^{D} .
$$

\section{Theoretical results}

In this section, we show that an implementation of PATHAVI in the original space enjoys the same properties as Theorem 2.1. We first identify sufficient conditions to allow a ray start. We define an implicit extreme point, which is a generalization of an extreme point when the lineality space is nontrivial, and show that there exists an implicit extreme point satisfying these sufficient conditions. A computational method for finding such an implicit extreme point is described in Section 4. Our conditions generalize those required for existing pivotal methods [6, 8, 19] for LCP, MCP, and AVI.

PATHAVI can process $L$-matrices with respect to the recession cone of the feasible set of the AVI. To this end, we show that a 1-manifold (the path $G_{C}^{-1}(0)$ ) generated by PATHAVI with a ray start at an implicit extreme point corresponds to a 1-manifold generated by the same pivotal method with a ray start at an extreme point in the reduced space. The reduced space is formed by projecting out the lineality space. This one-to-one correspondence is derived from the structural correspondence of the faces and the normal cones between the original space and the reduced one. Then by applying the existing processability result to the 1-manifold in the reduced space, we obtain the desired result.

\subsection{Sufficient conditions for a ray start and processability of PATHAVI}

We first identify sufficient conditions to perform a ray start at a point.

Proposition 3.1. Let an $\operatorname{AVI}(C, q, M)$ be given. If the following conditions are satisfied at a point $\bar{z}$, then we can perform a ray start at $\bar{z}$.

- $M \bar{z}+q \in \operatorname{aff}\left(N_{C}(\bar{z})\right)$.

- Every point in the interior of the $(n+1)$-cell $\left((\bar{z}+\operatorname{lin} C)+N_{C}(\bar{z})\right) \times \mathbb{R}_{+}$is regular.

- There exists a complementary basis at $\bar{z}$ such that aff $\left(N_{C}(\bar{z})\right)$ is spanned by columns of the basic variables in $(\lambda, w, v)$. 
Proof. Pick a vector $r \in \operatorname{ri}\left(N_{C}(\bar{z})\right)$. Let $(z, \lambda, w, v, s)$ be the complementary basic solution to (2) and (2) corresponding to the given complementary basis. Note that $z=\bar{z}$, thus $s$ is feasible. Therefore only basic variables in $(\lambda, w, v)$ might be infeasible. The first and third conditions say that we have $M z+q-A^{T} \lambda-w+$ $v=0$. By the third condition, for each $t \geq 0$ we have a unique $(\lambda(t), w(t), v(t))$ satisfying $M z+q-A^{T} \lambda(t)-$ $w(t)+v(t)-t r=0$. As $r \in \operatorname{ri}\left(N_{C}(\bar{z})\right)$, there exists $t^{0} \geq 0$ such that for all $t \geq t^{0}$ we have $M z+q-A^{T} \lambda(t)-$ $w(t)+v(t)-t r=0$ and $(\lambda(t), w(t), v(t))$ are feasible variables. Then for all $t \geq t^{0}(x(t), t)$ with $x(t):=$ $\bar{z}-A^{T} \lambda(t)-w(t)+v(t)$ lies in the cell $\left((\bar{z}+\operatorname{lin} C)+N_{C}(\bar{z})\right) \times \mathbb{R}_{+}$with $\pi_{C}(x(t))=\bar{z}$ and $G_{C}(x(t), t)=0$. By the second condition, the ray $(x(t), t)$ is generated at a regular point. By pivoting the $t$ variable into the complementary basis, we see that we can perform a ray start at $\bar{z}$.

Note that the sufficient conditions are satisfied at an extreme point. If $z$ is an extreme point, then aff $\left(N_{C}(z)\right) \equiv \mathbb{R}^{n}$ thus the first condition is trivially satisfied. Each extreme point has a corresponding basic feasible solution (BFS) to $A x-b=s$ [22, Section 3.4], and with that BFS we can construct a complementary basis satisfying the third condition as shown in Proposition 4.2 later in this paper. The second condition is also satisfied as proved in Proposition 3.4. As the existing pivotal methods [6, 8, 19] for LCP, MCP, and AVI perform a ray start at an extreme point, we see that the sufficient conditions generalize the existing result.

We now define an implicit extreme point, which is a generalization of an extreme point when the lineality space is nontrivial.

Definition 3.1. Let $C$ be a convex set in $\mathbb{R}^{n}$. A point $z \in C$ is called an implicit extreme point of $C$ if $z=\lambda z^{1}+(1-\lambda) z^{2}$ for any $z^{1}, z^{2} \in C$ and $\lambda \in(0,1)$ implies that $z-z^{1} \in \operatorname{lin} C$ and $z-z^{2} \in \operatorname{lin} C$.

Note that if the lineality space of $C$ is trivial, that is, lin $C=\{0\}$, then the definition of an implicit extreme point coincides with definition of an extreme point.

In the following four propositions, we study some characteristics of implicit extreme points. These characteristics are a generalization of those of extreme points. They are used as a tool for showing the existence of an implicit extreme point satisfying the sufficient conditions and for structural analysis later in this section. We start with faces consisting of only implicit extreme points. This generalizes 0-dimensional faces that are equivalent to extreme points. As the proof is elementary, we omit it.

Proposition 3.2. Let $C$ be a nonempty convex set in $\mathbb{R}^{n}$ and $\ell=\operatorname{dim}(\operatorname{lin} C)$. Then every point in an $\ell$ dimensional face of $C$ is an implicit extreme point of $C$. Also for each implicit extreme point $z$ of $C$ we have $F=z+\operatorname{lin} C$ is an $\ell$-dimensional face of $C$.

We prove next that the affine hull of the normal cone to $C$ at an implicit extreme point is the orthogonal complement of the lineality space of $C$. This generalizes the fact that the normal cone to $C$ at an extreme point is full-dimensional.

Proposition 3.3. A point $z$ is an implicit extreme point of a nonempty polyhedral convex set $C$ in $\mathbb{R}^{n}$ if and only if $z \in C$ and $\operatorname{aff}\left(N_{C}(z)\right)=(\operatorname{lin} C)^{\perp}$.

Proof. (only-if) Suppose that $z$ is an implicit extreme point of $C$. Using Proposition $3.2 F=z+\operatorname{lin} C$ is a face of $C$. We then have par $F=\operatorname{lin} C$. By [24, Proposition 2.1], par $F=\left(\operatorname{aff} N_{F}\right)^{\perp}$, where $N_{F}$ represents the normal cone having the same value for all $\hat{z} \in$ ri $F$, i.e., $N_{C}\left(\hat{z}^{1}\right)=N_{F}=N_{C}\left(\hat{z}^{2}\right)$ for all $\hat{z}^{1}, \hat{z}^{2} \in$ ri $F$. As $z \in$ ri $F$, it follows that aff $\left(N_{C}(z)\right)=(\operatorname{lin} C)^{\perp}$.

(if) Suppose that $z \in C$ and $\operatorname{aff}\left(N_{C}(z)\right)=(\operatorname{lin} C)^{\perp}$. Pick a face $F$ of $C$ such that $z \in$ ri $F$. Such a face exists by [26, Theorem 18.2]. Then $N_{C}(z)=N_{F}$, where $N_{F}$ is the normal cone having constant value on ri $F$. As par $F=\left(\operatorname{aff} N_{F}\right)^{\perp}$, we then have $\operatorname{par} F=\operatorname{lin} C$. Thus $F=z+\operatorname{lin} C$. By Proposition 3.2, $z$ is an implicit extreme point of $C$. 
Next we show that the second condition in Proposition 3.1 is satisfied at an implicit extreme point. Note that in the proposition below we show $\operatorname{dim}\left(M_{C}(\sigma)\right)=n$, which implies that $\operatorname{dim}\left(G_{C}\left(\sigma \times \mathbb{R}_{+}\right)\right)=n$.

Proposition 3.4. Let $z$ be an implicit extreme point of a nonempty polyhedral convex set $C$ in $\mathbb{R}^{n}$ and $\sigma$ be the cell $\left((z+\operatorname{lin} C)+N_{C}(z)\right)$ in the normal manifold of $C$. Then for an $A V I(C, q, M)$ with $M$ invertible on the lineality space of $C$, we have $\operatorname{dim}\left(M_{C}(\sigma)\right)=n$.

Proof. By [24, Proposition 2.5], $M_{C}$ coincides with some affine transformation $A_{\sigma}$ on $\sigma$. In the basis $Z=$ $(Q \bar{Q})$, we can represent the matrix $A_{\sigma}(\cdot)-A_{\sigma}(z)$ as follows:

$$
\left[\begin{array}{ll}
Q^{T} M Q & 0 \\
\bar{Q}^{T} M Q & I
\end{array}\right] .
$$

As $Q^{T} M Q$ is invertible, the matrix $A_{\sigma}(\cdot)-A_{\sigma}(z)$ is invertible. As $\sigma$ is $n$-dimensional, the result follows.

Finally, the last characteristic presents that on each $\ell$-dimensional face $F$ with $\ell=\operatorname{dim}(\operatorname{lin} C)$ (hence consisting of only implicit extreme points by Proposition 3.2 there exists an implicit extreme point $z \in F$ such that $M z+q \in$ aff $\left(N_{C}(z)\right)$. This generalizes the fact that at each extreme point $\bar{z}$ we have $M \bar{z}+q \in$ $\operatorname{aff}\left(N_{C}(\bar{z})\right) \equiv \mathbb{R}^{n}$.

Proposition 3.5. Let an $A V I(C, q, M)$ problem be given and $z \in C$ be an implicit extreme point of $C$. Assume that $M$ is invertible on the lineality space of $C$. Then there exists $\hat{z} \in z+\operatorname{lin} C$ such that $M \hat{z}+q \in \operatorname{aff}\left(N_{C}(\hat{z})\right)$.

Proof. For any implicit extreme point $\hat{z}$ of $C, M \hat{z}+q \in \operatorname{aff}\left(N_{C}(\hat{z})\right)$ if and only if $\pi_{\operatorname{lin} C}(M \hat{z}+q)=0$ by Proposition 3.3. By the assumption, $M_{Q Q}$ is invertible. Set

$$
\hat{z}=z+Q y \quad \text { where } \quad y=-M_{Q Q}^{-1}\left(Q^{T} q+M_{Q \bar{Q}} \bar{Q}^{T} z\right)-Q^{T} z
$$

Then $\hat{z} \in z+\operatorname{lin} C$ thus $\hat{z}$ is an implicit extreme point of $C$ by Proposition 3.2 , and

$$
\begin{aligned}
Q^{T}(M \hat{z}+q) & =Q^{T}\left(M\left[\begin{array}{ll}
Q & \bar{Q}
\end{array}\right]\left[\begin{array}{l}
Q^{T} \\
\bar{Q}^{T}
\end{array}\right] \hat{z}+q\right) \\
& =M_{Q Q}\left(Q^{T} \hat{z}\right)+M_{Q \bar{Q}}\left(\bar{Q}^{T} \hat{z}\right)+Q^{T} q \\
& =M_{Q Q}\left(Q^{T} z+y\right)+M_{Q \bar{Q}}\left(\bar{Q}^{T} z\right)+Q^{T} q \\
& =0
\end{aligned}
$$

It follows that $\pi_{\operatorname{lin} C}(M \hat{z}+q)=0$.

By Propositions 3.4 and 3.5, there exists an implicit extreme point satisfying the first two sufficient conditions for a ray start. We postpone checking the third condition to Section 4 as it requires a constructive proof. For the rest of this section, we assume that we have an implicit extreme point satisfying the sufficient conditions.

We now turn our attention to the processability of PATHAVI. Assume that we perform a ray start at an implicit extreme point and generate a 1-manifold in the original space $\mathbb{R}^{n}$. Our basic idea of deriving processability is that for this 1-manifold there corresponds to a 1-manifold generated by the same pivotal method with a ray start at an extreme point defined in the reduced space having possibly smaller dimension. We can then use the existing processability result [6, Theorem 4.4]. To establish the correspondence, we prove that there is a one-to-one correspondence between the faces, the normal cones, and the full-dimensional cells of the original space and reduced space as the following proposition shows. 
Proposition 3.6. Let $C$ be a nonempty polyhedral convex set in $\mathbb{R}^{n}$ and $\tilde{C}$ be the set $\tilde{C}=\bar{Q}^{T} C=\{\tilde{z} \mid \tilde{z}=$ $\bar{Q}^{T} z$ for some $\left.z \in C\right\}$ defined in $\mathbb{R}^{n-\ell}$ where $\ell=\operatorname{dim}(\operatorname{lin} C)$. Then the followings hold.

(a) $z$ is an implicit extreme point of $C$ if and only if $\tilde{z}=\bar{Q}^{T} z$ is an extreme point of $\tilde{C}$.

(b) $F$ is a face of $C$ if and only if $\tilde{F}=\bar{Q}^{T} F$ is a face of $\tilde{C}$.

(c) $v \in N_{C}(z)$ if and only if $v=\bar{Q} \tilde{v}$ for some $\tilde{v} \in N_{\tilde{C}}(\tilde{z})$ where $\tilde{z}=\bar{Q}^{T} z$.

(d) $\sigma$ is an $n$-cell of the normal manifold $\mathscr{N}_{C}$ of $C$ if and only if $\tilde{\sigma}=\bar{Q}^{T} \sigma$ is an $(n-\ell)$-cell of the normal manifold $\mathscr{N}_{\tilde{C}}$.

Proof. We prove in sequence. (a) (only-if) Let $z$ be an implicit extreme point of $C$. Set $\tilde{z}=\bar{Q}^{T} z$. We prove by contradiction. Suppose that $\exists \tilde{z}^{1}, \tilde{z}^{2} \in \tilde{C}$ and $\lambda \in(0,1)$ such that $\tilde{z}=\lambda \tilde{z}^{1}+(1-\lambda) \tilde{z}^{2}$ with $\tilde{z} \neq \tilde{z}^{i}$ for $i=1,2$. By definition of $\tilde{C}$, we have $z^{1}, z^{2} \in C$ such that $\tilde{z}^{i}=\bar{Q}^{T} z^{i}$ for $i=1,2$. As $C=\operatorname{lin} C \oplus\left((\operatorname{lin} C)^{\perp} \cap C\right)$ [26, page 65] and $\bar{Q}^{T} z=\bar{Q}^{T}\left(\lambda z^{1}+(1-\lambda) z^{2}\right)$, there exists $a \in \operatorname{lin} C$ such that $z=\lambda\left(a+z^{1}\right)+(1-\lambda)\left(a+z^{2}\right)$. As $\bar{Q}^{T}\left(z-\left(a+z^{i}\right)\right)=\tilde{z}-\tilde{z}^{i} \neq 0$, we have $z-\left(a+z^{i}\right) \notin \operatorname{lin} C$ for $i=1,2$, which contradicts our assumption that $z$ is an implicit extreme point of $C$.

(if) Using similar proof technique, we can show that for an extreme point $\tilde{z} \in \tilde{C} z$ is an implicit extreme point of $C$ when $\tilde{z}=\bar{Q}^{T} z$.

(b) (only-if) Let $F$ be a face of $C$. Set $\tilde{F}=\bar{Q}^{T} F$. Clearly, $\tilde{F}$ is a convex subset of $\tilde{C}$. Let $\tilde{z}^{1}, \tilde{z}^{2} \in \tilde{C}$ and $\lambda \in(0,1)$ satisfying $\lambda \tilde{z}^{1}+(1-\lambda) \tilde{z}^{2} \in \tilde{F}$. From $C=\operatorname{lin} C \oplus\left((\operatorname{lin} C)^{\perp} \cap C\right)$, we have $\bar{Q} \tilde{z}^{i} \in C$ for $i=1,2$. Then $\bar{Q}\left(\lambda \tilde{z}^{1}+(1-\lambda) \tilde{z}^{2}\right) \in F$ so that $\bar{Q} \tilde{z}^{1} \in F$ and $\bar{Q} \tilde{z}^{2} \in F$. This shows that $\tilde{z}^{i} \in \tilde{F}$ for $i=1,2$.

(if) Let $\tilde{F}=\bar{Q}^{T} F$ be a face of $\tilde{C}$. By the definition of $\tilde{F}, F$ is a convex subset of $C$. Let $z^{1}, z^{2} \in C$ and $\lambda \in(0,1)$ such that $\lambda z^{1}+(1-\lambda) z^{2} \in F$. We have $\bar{Q}^{T} z^{i} \in \tilde{C}$ for $i=1,2$ and $\bar{Q}^{T}\left(\lambda z^{1}+(1-\lambda) z^{2}\right) \in \tilde{F}$. Thus $\bar{Q}^{T} z^{i} \in \tilde{F}$, hence $z^{i} \in F+\operatorname{lin} C$ for $i=1,2$. Therefore $z^{i} \in F$ for $i=1,2$.

(c) For a vector $v \in \mathbb{R}^{n}$, we represent components of $v$ in $\operatorname{lin} C$ and $(\operatorname{lin} C)^{\perp}$ in the basis $\left[\begin{array}{ll}Q & \bar{Q}\end{array}\right]$ by $v_{Q}$ and $v_{\bar{Q}}$, respectively, so that $v=Q v_{Q}+\bar{Q} v_{\bar{Q}}$. If either $z \notin C$ or $\tilde{z} \notin \tilde{C}$, then we have nothing to prove. Therefore we assume that $z \in C$ and $\tilde{z} \in \tilde{C}$ in the proof. (only-if) Let $v \in N_{C}(z)$. By the definition of the normal cone, for each $a \in \operatorname{lin} C$ we have $\langle v,(z+a)-z\rangle \leq 0$ and $\langle v,(z-a)-z\rangle \leq 0$. Thus $\langle v, a\rangle=0$ for all $a \in \operatorname{lin} C$. Thus $N_{C}(z) \subset(\operatorname{lin} C)^{\perp}$ so that $v_{Q}=0$ and $v=\bar{Q} v_{\bar{Q}}$. We then have

$$
\begin{aligned}
0 & \geq\langle v, y-z\rangle, \quad \forall y \in C \\
& =\left\langle\bar{Q} v_{\bar{Q}}, Q y_{Q}+\bar{Q} y_{\bar{Q}}-\left(Q z_{Q}+\bar{Q} z_{\bar{Q}}\right)\right\rangle \\
& =\left\langle\bar{Q} v_{\bar{Q}}, \bar{Q}\left(y_{\bar{Q}}-z_{\bar{Q}}\right)\right\rangle \\
& =\left\langle v_{\bar{Q}}, y_{\bar{Q}}-z_{\bar{Q}}\right\rangle
\end{aligned}
$$

By setting $\tilde{v}=v_{\bar{Q}}, v=\bar{Q} \tilde{v}$ and $\tilde{v} \in N_{\tilde{C}}(\tilde{z})$.

(if) Let $\tilde{v} \in N_{\tilde{C}}(\tilde{z})$ and set $v=\bar{Q} \tilde{v}$. We have $\tilde{z}=\bar{Q}^{T} z$ if and only if $z \in \operatorname{lin} C+\bar{Q} \tilde{z}$. Let $z \in \operatorname{lin} C+\bar{Q} \tilde{z}$. Then

$$
\langle v, y-z\rangle=\left\langle\tilde{v}, y_{\bar{Q}}-z_{\bar{Q}}\right\rangle \leq 0, \quad y \in C
$$

The result follows.

(d) The result follows from (b), (c), and the definition of the full-dimensional cells of the normal manifold.

A similar result holds for the 1-manifold $G_{C}^{-1}(0)$. 
Proposition 3.7. Let an $\operatorname{AVI}(C, q, M)$ problem be given. Suppose that the matrix $M$ is invertible on the lineality space of $C$, and $G_{C}\left(x^{*}, t^{*}\right)=0$ where $r \in N_{C}\left(\pi_{C}\left(x^{0}\right)\right)$ for some $x^{0} \in \mathbb{R}^{n}$. Then the PL function $\tilde{G}_{\tilde{C}}(\tilde{x}, t):=\tilde{M} \pi_{\tilde{C}}(\tilde{x})+\tilde{q}+\tilde{x}-\pi_{\tilde{C}}(\tilde{x})-t \tilde{r}$ has value zero at $\left(\tilde{x}^{*}, t^{*}\right)$, where

$$
\begin{aligned}
\tilde{x}^{*} & =\bar{Q}^{T} x^{*} \\
Z & =\left[\begin{array}{ll}
Q & \bar{Q}
\end{array}\right], \\
\tilde{M} & =\left(Z^{T} M Z / M_{Q Q}\right)=M_{\bar{Q} \bar{Q}}-M_{\bar{Q} Q} M_{Q Q}^{-1} M_{Q \bar{Q}}, \\
\tilde{C} & =\bar{Q}^{T} C, \tilde{x}^{0}=\bar{Q}^{T} x^{0}, \tilde{q}=\left(\bar{Q}^{T}-M_{\bar{Q} Q} M_{Q Q}^{-1} Q^{T}\right) q, \\
\tilde{r} & =\bar{Q}^{T} r \in N_{\tilde{C}}\left(\pi_{\tilde{C}}\left(\tilde{x}^{0}\right)\right) .
\end{aligned}
$$

Conversely, if $\tilde{G}_{\tilde{C}}\left(\tilde{x}^{*}, t^{*}\right)=0$ then $G_{C}\left(x^{*}, t^{*}\right)=0$ with $x^{*}=\bar{Q} \tilde{x}^{*}+Q y^{*}$ and

$$
y^{*}=-M_{Q Q}^{-1}\left(M_{Q \bar{Q}} \pi_{\tilde{C}}\left(\tilde{x}^{*}\right)+Q^{T} q\right) .
$$

Proof. Let $\left(x^{*}, t^{*}\right)$ satisfying $G_{C}\left(x^{*}, t^{*}\right)=0$ with $r \in N_{C}\left(\pi_{C}\left(x^{0}\right)\right)$ for some $x^{0}$ be given. Then

$$
\begin{gathered}
M \pi_{C}\left(x^{*}\right)+q+x^{*}-\pi_{C}\left(x^{*}\right)-t^{*} r=0, \\
(\Rightarrow)\left[\begin{array}{c}
Q^{T} \\
\bar{Q}^{T}
\end{array}\right] M\left[\begin{array}{ll}
Q & \bar{Q}
\end{array}\right]\left[\begin{array}{l}
Q^{T} \\
\bar{Q}^{T}
\end{array}\right] \pi_{C}\left(x^{*}\right)+\left[\begin{array}{c}
Q^{T} \\
\bar{Q}^{T}
\end{array}\right]\left(q+x^{*}-\pi_{C}\left(x^{*}\right)-t^{*} r\right)=0, \\
(\Rightarrow)\left[\begin{array}{cc}
M_{Q Q} & M_{Q \bar{Q}} \\
M_{\bar{Q} Q} & M_{\bar{Q} \bar{Q}}
\end{array}\right]\left[\begin{array}{c}
Q^{T} \pi_{C}\left(x^{*}\right) \\
\bar{Q}^{T} \pi_{C}\left(x^{*}\right)
\end{array}\right]+\left[\begin{array}{c}
Q^{T} q \\
\bar{Q}^{T}\left(q+x^{*}-\pi_{C}\left(x^{*}\right)-t^{*} r\right)
\end{array}\right]=0, \\
(\Rightarrow) \tilde{M} \bar{Q}^{T} \pi_{C}\left(x^{*}\right)+\tilde{q}+\bar{Q}^{T}\left(x^{*}-\pi_{C}\left(x^{*}\right)\right)-t^{*} \tilde{r}=0, \\
\quad \operatorname{using} Q^{T} \pi_{C}\left(x^{*}\right)=-M_{Q Q}^{-1}\left(M_{Q \bar{Q}} \bar{Q}^{T} \pi_{C}\left(x^{*}\right)+Q^{T} q\right), \\
(\Rightarrow) \tilde{M} \pi_{\tilde{C}}\left(\tilde{x}^{*}\right)+\tilde{q}+\tilde{x}^{*}-\pi_{\tilde{C}}\left(\tilde{x}^{*}\right)-t^{*} \tilde{r}=0 .
\end{gathered}
$$

The second $(\Rightarrow)$ holds because $N_{C}\left(\pi_{C}\left(x^{0}\right)\right) \subset(\text { lin } C)^{\perp}$. The last $(\Rightarrow)$ holds because $\bar{Q}^{T} \pi_{C}(x)=\pi_{\bar{Q}^{T} C}\left(\bar{Q}^{T} x\right)$ by [5, Lemma 2.1]. Also $\tilde{r} \in N_{\tilde{C}}\left(\pi_{\tilde{C}}\left(\tilde{x}^{0}\right)\right)$ by Proposition 3.6 d).

Conversely, let $\tilde{G}_{\tilde{C}}\left(\tilde{x}^{*}, t^{*}\right)=0$. Set $x^{*}=\bar{Q} \tilde{x}^{*}+Q y^{*}$ with $y^{*}$ as specified in the proposition. Then

$$
\begin{aligned}
\pi_{C}\left(x^{*}\right) & =\pi_{C \cap(\operatorname{lin} C)^{\perp}}\left(x^{*}\right)+\pi_{\operatorname{lin} C}\left(x^{*}\right) \\
& =\bar{Q} \pi_{\tilde{C}}\left(\tilde{x}^{*}\right)+Q y^{*}
\end{aligned}
$$

Therefore $Q^{T} \pi_{C}\left(x^{*}\right)=y^{*}$. By the definition of $y^{*}$, the converse directions also hold.

Note that the $\operatorname{AVI}(\tilde{C}, \tilde{q}, \tilde{M})$ with $\tilde{C}, \tilde{q}$, and $\tilde{M}$ as in Proposition 3.7 is the same exact problem obtained by applying the stage 1 reduction [6, page 49] to the $\operatorname{AVI}(C, q, M)$. Also $\tilde{G}_{\tilde{C}}$ is the PL function defined on the $(n-\operatorname{dim}(\operatorname{lin} C)+1)$-manifold $\mathscr{M}_{\tilde{C}}$ of $\tilde{C}$ to find a zero of the normal map associated with the $\operatorname{AVI}(\tilde{C}, \tilde{q}, \tilde{M})$.

An implication of Proposition 3.7 is that if $G_{C}(x+\theta \Delta x, t+\theta \Delta t)=0$ and $(x+\theta \Delta x, t+\theta \Delta t) \in \sigma \times \mathbb{R}_{+}$ for all $\theta \in[0, v]$ for some $v>0$, possibly $v=\infty$, and $\sigma \times \mathbb{R}_{+}$is an $(n+1)$-cell of $\mathscr{M}_{C}$, then we have $\tilde{G}_{\tilde{C}}(\tilde{x}+\theta \Delta \tilde{x}, t+\theta \Delta t)=0$ with $(\tilde{x}+\theta \Delta \tilde{x}, t+\theta \Delta t) \in \tilde{\sigma} \times \mathbb{R}_{+}$for all $\theta \in[0, v]$, where $\tilde{\sigma}=\bar{Q}^{T} \sigma$ and $\Delta \tilde{x}=$ $\bar{Q}^{T} \Delta x$. The converse also holds by setting $\Delta x=\bar{Q} \Delta \tilde{x}+Q \Delta y$ with $\Delta y=-M_{Q Q}^{-1} M_{Q \bar{Q}} H_{\tilde{\sigma}} \Delta \tilde{x}$, where $H_{\tilde{\sigma}}$ is a matrix representing the projection operator $\pi_{\tilde{C}}(\cdot)$ on elements of $\tilde{\sigma}$. Therefore the projection of each piece 
of $G_{C}^{-1}(0)$ onto $\mathscr{M}_{\tilde{C}}$ corresponds to each piece of $\tilde{G}_{\tilde{C}}^{-1}(0)$ and vice versa. As a consequence, if $G_{C}^{-1}(0)$ contains a ray, i.e., $\exists(\Delta x, \Delta t) \neq 0$ with $v=\infty$ on some $(n+1)$-cell $\sigma \times \mathbb{R}_{+}$of $\mathscr{M}_{C}$, and the corresponding value $(\Delta \tilde{x}, \Delta t)$ is not zero, then the corresponding piece of $\tilde{G}_{\tilde{C}}^{-1}(0)$ is also a ray. The following proposition shows that whenever there is a ray in $G_{C}^{-1}(0)$ with $\Delta x \neq 0$, then we have $\Delta \tilde{x} \neq 0$ so that the corresponding piece of $\tilde{G}_{\tilde{C}}^{-1}(0)$ is also a ray. Note that the converse automatically holds as $\Delta x \neq 0$ for each $\Delta \tilde{x} \neq 0$.

Proposition 3.8. For an $A V I(C, q, M)$, suppose that PATHAVI generates $G_{C}^{-1}(0)$ with a ray start at an implicit extreme point. For each ray in $G_{C}^{-1}(0)$ in the direction of $(\Delta x, \Delta t) \neq 0$, if $\Delta x \neq 0$ then $\Delta \tilde{x}:=\bar{Q}^{T} \Delta x$ is a ray in $\tilde{G}_{\tilde{C}}^{-1}(0)$ that is nonzero under the assumption that either 0 is a regular value or we do lexicographic pivoting.

Proof. Let $z$ be an implicit extreme point at which PATHAVI performs a ray start. By construction, $\Delta \tilde{x}=0$ if and only if $\Delta x \in \operatorname{lin} C$. For the starting ray we have $\Delta x \in N_{C}(z)$ so that $\Delta x \notin \operatorname{lin} C$ by Proposition 3.3. Thus $\Delta \tilde{x} \neq 0$.

We now assume that there is a ray in $G_{C}^{-1}(0)$ other than the starting ray. Suppose that $\Delta x \in \operatorname{lin} C$. We prove by contradiction. Let us assume that the ray is generated at the $(k+1)$ th iteration of complementary pivoting, and it starts from $x^{k+1}$. We know that $x^{k+1} \in \sigma^{k+1} \times \mathbb{R}_{+}$and $x^{k+1} \in \sigma^{k} \times \mathbb{R}_{+}$, where $\sigma^{k} \times \mathbb{R}_{+}$is the $(n+1)$-cell of $\mathscr{M}_{C}$ PATHAVI passes through at the $k$ th complementary pivoting iteration. As $\operatorname{lin} C \subset \operatorname{lin} \sigma$ for each $(n+1)$-cell $\sigma \times \mathbb{R}_{+}$of $\mathscr{M}_{C}, x^{k+1}+\theta \Delta x \in \sigma^{k}$ for all $\theta \geq 0$. This contradicts the fact that $G_{C}^{-1}(0)$ is a 1-manifold neat in $\mathscr{M}_{C}$ [12, Theorem 9.1 or Lemma 15.5], that is, $G^{-1}(0) \cap\left(\sigma^{k} \times \mathbb{R}_{+}\right)$must be expressed as an intersection of $\sigma^{k} \times \mathbb{R}_{+}$with a line. Therefore $\Delta x \notin \operatorname{lin} C$. The result follows.

From Lemma7.1, if $M$ is semimonotone with respect to $\operatorname{rec} C$ and invertible on lin $C$, we have $\Delta t=0$ whenever PATHAVI generates a ray in the direction of $(\Delta x, \Delta t)$. These classes include the $L$-matrix class and the new matrix classes defined in Section 3.2 Therefore whenever PATHAVI generates a ray in $G_{C}^{-1}(0)$ for those classes of matrices the corresponding piece in $\tilde{G}_{\tilde{C}}^{-1}(0)$ is also a ray by Proposition 3.8

With Propositions 3.6-3.8, we finally prove that PATHAVI can process $L$-matrices as defined in [6, Theorem 4.4]. In contrast to [6], the following proof does not apply any reduction to achieve the result.

Theorem 3.1. Suppose that $C$ is a polyhedral convex set, and $M$ is an L-matrix with respect to rec $C$ which is invertible on the lineality space of $C$. Then exactly one of the following occurs:

- PATHAVI solves the AVI $(C, q, M)$.

- The following system has no solution

$$
M z+q \in(\operatorname{rec} C)^{D} .
$$

Proof. By Propositions 3.6 3.7 for a 1-manifold $G_{C}^{-1}(0)$ generated by PATHAVI there corresponds to a 1-manifold $\tilde{G}_{\tilde{C}}^{-1}(0)$ in the reduced space generated by the same pivotal method with a ray start at an extreme point of $\tilde{C}$ with $\tilde{M}$ an $L$-matrix with respect to rec $\tilde{C}$. If there is a secondary ray in $G_{C}^{-1}(0)$, then so is in $\tilde{G}_{\tilde{C}}^{-1}(0)$ by Proposition 3.8 Therefore there exists directions $(\Delta \tilde{x}, \Delta \tilde{z}, \Delta \tilde{\lambda}, \Delta \tilde{s}, \Delta t)$ in the reduced space satisfying

$$
\begin{aligned}
\Delta \tilde{x}-\Delta \tilde{z} & =-\tilde{M} \Delta \tilde{z}+r \Delta t, \\
A_{\mathscr{A} \bullet} \Delta \tilde{z} & =0, \\
A_{\mathscr{A} \bullet} \Delta \tilde{z}-\Delta \tilde{s}_{\mathscr{A}} & =0, \\
\Delta \tilde{x}-\Delta \tilde{z} & =-A_{\mathscr{A} \bullet}^{T} \Delta \tilde{\lambda}_{\mathscr{A}}
\end{aligned}
$$


where we have included bound constraints in the matrix $A$ for clarity, and $\mathscr{A}$ and $\bar{A}$ denote the active and inactive sets, respectively. We then apply Theorem 2.1 to (4) to get the desired result.

\subsection{Additional processability results}

Let us now extend the classes of AVIs that PATHAVI is able to process. The results in Lemmas 3.1 3.2 consider the structure of the whole AVI, not only $M$ and $C$. As stated previously, a 1-manifold generated in $\mathscr{M}_{C}$ corresponds to another one in $\mathscr{M}_{\tilde{C}}$. Hence, in the following we denote by $\operatorname{AVI}(\tilde{C}, \tilde{q}, \tilde{M})$ the AVI corresponding to $\operatorname{AVI}(C, q, M)$ with the lineality space projected out. If $M$ is invertible on lin $C$, the results can then be applied to original AVI by noting that the projections of the directions of the rays on $G_{C}^{-1}(0)$ are solution to the system of equations (4) in the reduced space.

In Section 6.1, we present a friction contact problem where Theorem 3.1 cannot be applied but the following lemma can.

Lemma 3.1. Consider an $A V I(\tilde{C}, \tilde{q}, \tilde{M})$ with lin $\tilde{C}=\{0\}$. Suppose that $\tilde{M}$ is semimonotone with respect to rec $\tilde{C}$ and that for any solution $z \neq 0$ of the problem

$$
z \in \operatorname{rec} \tilde{C}, \quad \tilde{M} z \in(\operatorname{rec} \tilde{C})^{D}, \quad z^{T} \tilde{M} z=0,
$$

it holds that

$$
z^{T}\left(\tilde{M} z^{\prime}+\tilde{q}\right) \geq 0, \quad \forall z^{\prime} \in \tilde{C} .
$$

Then PATHAVI solves the AVI $(\tilde{C}, \tilde{q}, \tilde{M})$.

Proof. The pivotal method used in PATHAVI fails if an unbounded ray is generated at some iterate $\left(x^{k}, t^{k}\right)$, $k>0$. Now suppose that the method generates an unbounded ray. From Lemma 7.1 we know that $\Delta t=0$, and $\Delta z \neq 0$ is a solution to $(5)$. This means that for any point $x^{k+1}$ on the ray, we have $\tilde{G}_{\tilde{C}}\left(x^{k+1}, t^{k}\right)=0$, implying that

$$
\left\langle\Delta z, \tilde{G}_{\tilde{C}}\left(x^{k+1}, t^{k}\right)\right\rangle=\left\langle\Delta z, \tilde{M} z^{k+1}+\tilde{q}\right\rangle+\left\langle\Delta z, x^{k+1}-z^{k+1}\right\rangle+\left\langle\Delta z,-t^{k} r\right\rangle=0
$$

The first term is non-negative by our assumption, as well as the second one by the normal cone definition. The third one is strictly positive since $-t^{k} r \in \operatorname{int}(\operatorname{rec} \tilde{C})^{D}$. Hence we reached a contradiction.

An additional property on $\tilde{M}$ allows easier checking of the conditions 6 of Lemma 3.1

Corollary 3.1. If for any solution $z$ of $(5)$ we have $\left\langle z^{\prime}, \tilde{M}^{T} z\right\rangle \geq 0$, for all $z^{\prime} \in \tilde{C}$, then the condition (6) reduces to $z^{T} \tilde{q} \geq 0$ whenever $z$ is a solution to $(5)$.

We introduce an additional problem class PATHAVI can process.

Lemma 3.2. Consider an $A V I(\tilde{C}, \tilde{q}, \tilde{M})$ with $\operatorname{lin} \tilde{C}=\{0\}$. Suppose that $\tilde{C}$ is a proper cone, $\tilde{M}$ is copositive with respect to $\tilde{C}$ and that the following implication holds:

$$
z \in \operatorname{rec} \tilde{C}, \quad \tilde{M} z \in(\operatorname{rec} \tilde{C})^{D}, \quad z^{T} \tilde{M} z=0 \quad \Rightarrow \quad z^{T} \tilde{q} \geq 0 .
$$

Then the AVI $(\tilde{C}, \tilde{q}, \tilde{M})$ has a solution and PATHAVI finds it. 
Proof. Recall from [6, Lemma 4.3], that a copositive matrix is also semimonotone. This implies that $\Delta t=0$ and that $\Delta z \neq 0$ satisfies the left-hand side of (7). Now let us suppose that at the current iterate $x_{k}$, there exists an unbounded ray. Letting $z_{k+1}=z_{k}+\theta \Delta z$ and computing the inner product $\left\langle z_{k+1}, \tilde{G}_{\tilde{C}}\left(x_{k+1}, t_{k}\right)\right\rangle$ yields

$$
0=\left\langle z_{k+1}, \tilde{G}_{\tilde{C}}\left(x_{k+1}, t_{k}\right)\right\rangle=\left\langle z_{k+1}, \tilde{M} z_{k+1}\right\rangle+\left\langle z_{k+1}, \tilde{q}\right\rangle+\left\langle z_{k+1}, x_{k+1}-z_{k+1}\right\rangle+\left\langle z_{k+1},-t_{k} r\right\rangle .
$$

Note that since $\tilde{C}$ is pointed, $\left\langle z_{k+1}, x_{k+1}-z_{k+1}\right\rangle \geq 0$ by the definition of the normal cone. The first term is quadratic in $\theta$ while the second and third are linear in $\theta$. Therefore, if $\langle\Delta z, \tilde{M} \Delta z\rangle>0$, then $\left\langle z_{k+1}, \tilde{G}_{\tilde{C}}\left(x_{k+1}, t_{k}\right)\right\rangle>$ 0 for $\theta$ large enough and we reach a contradiction. We are left with the case $\langle\Delta z, \tilde{M} \Delta z\rangle=0$ :

$$
0=\left\langle z_{k}, \tilde{q}\right\rangle-\left\langle z_{k}, t_{k} r\right\rangle+\left\langle z_{k+1}, x_{k+1}-z_{k+1}\right\rangle+\left\langle z_{k+1}, \tilde{M} z_{k+1}\right\rangle+\theta\left(\langle\Delta z, \tilde{q}\rangle+\left\langle\Delta z,-t_{k} r\right\rangle\right) .
$$

The sum multiplied by $\theta$ is positive since $-t_{k} r \in \operatorname{int}(\operatorname{rec} \tilde{C})^{D}$. Now the first two terms are constant and the third and fourth ones are nonnegative. Whence for $\theta$ large enough, $\left\langle z_{k+1}, \tilde{G}_{\tilde{C}}\left(x_{k+1}, t_{k}\right)\right\rangle$ is positive, which concludes the proof.

Remark. Lemma 3.2 was already known for the LCP case (that is $\tilde{C}=\mathbb{R}_{+}^{n}$ ): the existence of a solution is given in [7. Theorem 3.8.6]. Here we are able to provide a constructive proof for an AVI $(\tilde{C}, \tilde{q}, \tilde{M})$.

Let us present an $\operatorname{AVI}(C, q, M)$ that satisfies the conditions of Lemma 3.2 where $M$ is not an $L$-matrix. Suppose that $C \subseteq \mathbb{R}_{+}^{n+1}$ is a polyhedral solid cone, $M=\left(\begin{array}{cc}I_{n} & 0 \\ \mathbf{1}_{n}^{T} & 0\end{array}\right)$, with $\mathbf{1}_{n}$ the vector of ones of size $n$ and $q=\left(0_{n}, 1\right)^{T}$. The solution set of the system $x \in C, M x=0$ and $x^{T} M x=0$ is $\left\{\left(0_{n}, \alpha\right)^{T}, \alpha \geq 0\right\}$. Note that if $x=\left(0_{n}, \alpha\right)^{T}, \alpha>0$, then $A x=0$ and $x^{T} M x=0$. However, for any nonzero vector $x^{\prime}=\left(x_{1}^{\prime T}, \alpha^{\prime}\right)^{T}$ in $C$, $-M^{T} x^{\prime}=\left(-I_{n} x_{1}^{\prime T}-\alpha^{\prime} \mathbf{1}^{T}, 0\right)^{T} \notin C^{D}$. Therefore, condition (b) of the $L$-matrix fails to hold. On the other hand, we can readily check that $M$ is copositive with respect to $C$ and that for any $x=\left(0_{n}, \alpha\right)^{T}, \alpha \geq 0$, $x^{T} q=\alpha \geq 0$. Whence Lemma 3.2 can be used.

\section{Computing an implicit extreme point for a ray start}

In this section, we describe how to compute an implicit extreme point satisfying the sufficient conditions for a ray start and the complementary basis associated with it so that we can start complementary pivoting at that implicit extreme point. An implicit extreme point is computed using a linear programming (LP) solver, i.e., CPLEX or GUROBI, with possibly additional pivoting, and its complementary basis is computed based on the basis information given by the LP solver. The use of the existing LP solver, which has fast sparse linear algebra engine and pivoting method, as well as the use of sparse linear algebra engine for complementary pivoting enables PATHAVI to fully exploit the sparse representation of the given AVI. This makes our method efficient for large-scale AVI problems. See for example Section 6.2.

We start with an introduction to some terminology and notational conventions for describing a basic solution of an LP problem. We follow notation used in [4]. Suppose that we run an LP solver over an LP problem: minimize $c^{T} z$ subject to $A z-b \in K$ and $l \leq z \leq u$. Without loss of generality, we assume that we have eliminated all fixed variables. For each solution $z$ obtained from the LP solver, we have four index sets, $B, N_{l}, N_{u}$, and $N_{f r}$, for variables and two index sets, $\mathscr{A}$ and $\overline{\mathscr{A}}$, for constraints described by $A$ and $b .11$ Table 4 in the Appendix lists the properties of the index sets and the solution $z$. In Table 4, if $l_{B} \leq z_{B} \leq u_{B}$, we say that $z$ is a basic feasible solution. Otherwise, we say that $z$ is a basic solution. Note that we have $|\mathscr{A}|=|B|$ in Table 4 as the basis matrix $\mathbf{B}$ is invertible. Hence the submatrix $A_{\mathscr{A} B}$ of $\mathbf{B}$ is square and invertible.

\footnotetext{
${ }^{1}$ These index sets can be obtained using CPXgetbase() for CPLEX, for example.
} 
We first describe how to compute an implicit extreme point of $C$, based on which we compute another implicit extreme point if necessary satisfying the sufficient conditions for a ray start. For a given $\operatorname{AVI}(C, q, M)$, we formulate and solve the following LP problem using an LP solver:

$$
\begin{array}{ll}
\text { minimize } & 0^{T} z \\
\text { subject to } & A z-b \in K \\
& l \leq z \leq u
\end{array}
$$

We put zero objective coefficients in the $(\mathrm{LP})$ so that the $(\mathrm{LP})$ returns right away once it finds a basic feasible solution; the (LP) returns once it solves the phase I of the simplex method. If we have a good knowledge about where to start complementary pivoting, then we could try to solve the (LP) with different objective coefficients.

Assuming that the $(\mathrm{LP})$ is feasible, a basic feasible solution $z^{0}$ from the LP solver with the corresponding index sets is an extreme point if $N_{f r}=\varnothing$. When $N_{f r} \neq \varnothing, z^{0}$ might not be an implicit extreme point. In this case, we move from $z^{0}$ to another implicit extreme point by doing additional pivoting in a way that we make as many nonbasic free variables as basic variables. Algorithm 1 in the Appendix describes the pivoting procedure. After applying Algorithm 1 , for each $j \in N_{f r}$ and $d^{j}=A_{\mathscr{A} B}^{-1} A_{\mathscr{A}, j}$ if there exists $k$ such that $d_{k}^{j} \neq 0$, then the basic variable corresponding to the $k$ th position in $B$ is a free variable. Otherwise, the variable $z_{j}$ must have been pivoted in by Algorithm 1 . Also note that Algorithm 1 doesn't change the properties described in Table 4 Using Algorithm 1, we obtain the following result.

Proposition 4.1. Suppose that we have applied Algorithm 1$]$ Then the new point, denoted by $z^{0}$, constructed from $z^{0}$ through Algorithm 1 is an implicit extreme point of $C$. We have $\operatorname{dim}(\operatorname{lin} C)=\left|N_{f r}\right|$ and the following set of vectors is a basis for the lineality space of $C$ :

$$
\bigcup_{j \in N_{f r}}\left\{v^{j}\right\}, \quad v_{k}^{j}= \begin{cases}\left(A_{\mathscr{A} B}^{-1} A_{\mathscr{A}, j}\right)_{k} & \text { if } k \in B, \\ 0 & \text { if } k \in N_{l} \cup N_{u}, \\ 0 & \text { if } k \in N_{f r}, k \neq j, \\ 1 & \text { if } k=j .\end{cases}
$$

Proof. Clearly, $\bar{z}^{0} \in C$ as we do a ratio test to move the point. We first show that lin $C=\left|N_{f r}\right|$ and $\left\{v^{j}\right\}_{j \in N_{f r}}$ is a basis for the lineality space of $C$. For each $j \in N_{f r}$, if $v_{k}^{j} \neq 0$ for $k \in B$, then we have $l_{k}=-\infty$ and $u_{k}=\infty$ as discussed in the previous paragraph. It follows that $z^{0}+\lambda v^{j} \in C$ for all $\lambda \in \mathbb{R}$. By [26, Theorem 8.3], $v^{j} \in \operatorname{rec} C \cap(-\operatorname{rec} C)$. Thus $v^{j} \in \operatorname{lin} C$. By construction of $v^{j}$, we see that $v^{j}$ 's are linearly independent. This implies that $\operatorname{dim}(\operatorname{lin} C) \geq\left|N_{f r}\right|$. As $\operatorname{dim}\left(N_{C}\left(\bar{z}^{0}\right)\right) \geq|B|+\left|N_{l}\right|+\left|N_{u}\right|$ and $N_{C}\left(\bar{z}^{0}\right) \subset(\operatorname{lin} C)^{\perp}$ as shown in Proposition 3.6(d), it follows that $\operatorname{dim}(\operatorname{lin} C)=\left|N_{f r}\right|$ and $\left\{v^{j}\right\}_{j \in N_{f r}}$ is a basis for the lineality space of $C$.

We now prove that $\bar{z}^{0}$ is an implicit extreme point of $C$. Suppose that $z^{0}=\lambda z^{1}+(1-\lambda) z^{2}$ for some $z^{1}, z^{2} \in C$ and $\lambda \in(0,1)$. Define $d^{k}=\sum_{j \in N_{f r}}\left(-z_{j}^{k} v^{j}\right)$ and set $\tilde{z}^{k}=z^{k}+d^{k}$ for $k=1,2$. We then have $\tilde{z}_{j}^{k}=0$ for $j \in N_{f r}$ and $\tilde{z}^{k} \in C$ as $d^{k} \in \operatorname{lin} C$ for $k=1,2$. As $\bar{z}^{0}=\lambda z^{1}+(1-\lambda) z^{2}, \bar{z}^{0}=\lambda \tilde{z}^{1}+(1-\lambda) \tilde{z}^{2}-\left(\lambda d^{1}+\right.$ $\left.(1-\lambda) d^{2}\right)$. We have $\lambda d^{1}+(1-\lambda) d^{2}=\sum_{j \in N_{f r}}\left(-\left(\lambda z_{j}^{1}+(1-\lambda) z_{j}^{2}\right) v^{j}\right)$. As $\bar{z}_{N_{f r}}^{0}=\tilde{z}_{N_{f r}}^{1}=\tilde{z}_{N_{f r}}^{2}=0, v_{j}^{j}=1$, and $v_{h}^{j}=0$ for $h \in N_{f r}, h \neq j$, we see that $\lambda d^{1}+(1-\lambda) d^{2}=0$. Therefore, $\bar{z}^{0}=\lambda \tilde{z}^{1}+(1-\lambda) \tilde{z}^{2}$. It follows that $\bar{z}^{0}=\tilde{z}^{1}=\tilde{z}^{2}$. Thus, $\bar{z}^{0}-z^{k}=d^{k} \in \operatorname{lin} C$ for $k=1,2$, which implies that $\bar{z}^{0}$ is an implicit extreme point of $C$.

With the implicit extreme point $\bar{z}^{0}$ of $C$ and the index sets $\left(B, N_{l}, N_{u}, N_{f r}, \mathscr{A}, \bar{A}\right)$ associated with it, we finally construct an initial complementary basis and compute an implicit extreme point satisfying the 
sufficient conditions for a ray start using that complementary basis. To prove the invertibility of our initial complementary basis, we first need to introduce the following technical result derived from [20, Lemma 3.6].

Corollary 4.1. Suppose that we have index sets $\left(B, N_{l}, N_{u}, N_{f r}, \mathscr{A}, \mathscr{A}\right)$ associated with an $A V I(C, q, M)$ with a nonempty $N_{f r}$. Then $Z$ is invertible if and only if $\tilde{W}^{T} \tilde{M} \tilde{W}$ is invertible, where

$$
Z=\left[\begin{array}{ccc}
M_{B B} & M_{B N_{f r}} & -A_{\mathscr{A} B}^{T} \\
M_{N_{f r} B} & M_{N_{f r} N_{f r}} & -A_{\mathscr{A} N_{f r}}^{T} \\
A_{\mathscr{A} B} & A_{\mathscr{A} N_{f r}} & 0
\end{array}\right], \quad \tilde{M}=\left[\begin{array}{cc}
M_{B B} & M_{B N_{f r}} \\
M_{N_{f r} B} & M_{N_{f r} N_{f r}}
\end{array}\right], \quad \tilde{W}=\left[\begin{array}{c}
-A_{\mathscr{A} B}^{-1} A_{\mathscr{A} N_{f r}} \\
I_{N_{f r}}
\end{array}\right],
$$

and $I_{N_{f r}}$ is an identity matrix of size $\left|N_{f r}\right| \times\left|N_{f r}\right|$.

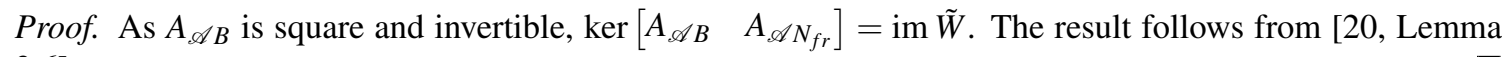
3.6].

We are now ready to present our initial complementary basis and an implicit extreme point satisfying the sufficient conditions for a ray start.

Proposition 4.2. For a given $\operatorname{AVI}(C, q, M)$, suppose that we have an implicit extreme point $\bar{z}^{0}$ and the index sets $\left(B, N_{l}, N_{u}, N_{f r}, \mathscr{A}, \mathscr{A}\right)$ associated with $\bar{z}^{0}$. Then the matrix on the left-hand side of the following system of equations is invertible if and only if $M$ is invertible on the lineality space of C. Also $z=\left(z_{B}, z_{N_{f r}}, z_{N_{l}}^{0}, z_{N_{u}}^{0}\right)$ in a solution to the system of equations satisfies $z \in z^{0}+\operatorname{lin} C$, i.e., $z$ is an implicit extreme point of $C$ by Proposition 3.2 in Section 3 and $M z+q \in$ aff $\left(N_{C}(z)\right)$.

$$
\left[\begin{array}{cccccc}
M_{B B} & M_{B N_{f r}} & -A_{\mathscr{A} B}^{T} & 0 & 0 & 0 \\
M_{N_{l} B} & M_{N_{l} N_{f r}} & -A_{\mathscr{A} N_{l}}^{T} & -I_{N_{l}} & 0 & 0 \\
M_{N_{u} B} & M_{N_{u} N_{f r}} & -A_{\mathscr{A} N_{u}}^{T} & 0 & I_{N_{u}} & 0 \\
M_{N_{f r} B} & M_{N_{f r} N_{f r}} & -A_{\mathscr{A} N_{f r}}^{T} & 0 & 0 & 0 \\
A_{\mathscr{A} B} & A_{\mathscr{A} N_{f r}} & 0 & 0 & 0 & 0 \\
A_{\mathscr{A} B} & A_{\mathscr{A} N_{f r}} & 0 & 0 & 0 & -I_{\mathscr{A}}
\end{array}\right]\left[\begin{array}{c}
z_{B} \\
z_{N_{f r}} \\
\lambda_{\mathscr{A}} \\
w_{N_{l}} \\
v_{N_{u}} \\
s_{\mathscr{A}}
\end{array}\right]=\left[\begin{array}{c}
-q_{B}-M_{B N} \bar{z}_{N}^{0} \\
-q_{N_{l}}-M_{N_{l} N} \bar{z}_{N}^{0} \\
-q_{N_{u}}-M_{N_{u} N} \bar{z}_{N}^{0} \\
-q_{N_{f r}}-M_{N_{f r} N} \bar{z}_{N}^{0} \\
b_{\mathscr{A}}-A_{\mathscr{A} N} z_{N}^{0} \\
b_{\mathscr{A}}-A_{\mathscr{A} N} \bar{z}_{N}^{0}
\end{array}\right] .
$$

Proof. The matrix on the left-hand side of the system of equations is invertible if and only if the matrix $Z$ defined in Corollary 4.1 is invertible. This is because of the identity submatrices of it, $-I_{N_{l}}, I_{N_{u}}$, and $-I_{\mathscr{A}}$. Define $W=\left(\begin{array}{llll}-A_{\mathscr{A} B}^{-1} A_{\mathscr{A} N} N_{f r} & I_{N_{f r}} & 0_{N_{l}} & 0_{N_{u}}\end{array}\right)^{T}$ where $I_{N_{f r}}$ is an identity matrix of size $\left|N_{f r}\right| \times\left|N_{f r}\right|$, and $0_{N_{l}}$ and $0_{N_{u}}$ are zero matrices of sizes $\left|N_{l}\right| \times\left|N_{f r}\right|$ and $\left|N_{u}\right| \times\left|N_{f r}\right|$, respectively. We see that the columns of $W$ is a basis for the lineality space of $C$. We then have $W^{T} M W=\tilde{W} \tilde{M}^{T} \tilde{W}$ where $\tilde{W}$ and $\tilde{M}$ are the matrices defined in Corollary 4.1. Therefore, the matrix is invertible if and only if $M$ is invertible on the lineality space of $C$.

We now show that a $z$-part solution $z$ to the system of equations satisfies $z \in \bar{z}^{0}+\operatorname{lin} C$. From the system of equations, we have

$$
z_{B}=-A_{\mathscr{A} B}^{-1} A_{\mathscr{A} N_{f r}} z_{N_{f r}}+A_{\mathscr{A} B}^{-1}\left(b_{\mathscr{A}}-A_{\mathscr{A} N} \bar{z}_{N}^{0}\right) .
$$

If $z_{N_{f r}}=0$ at a solution, then $z_{B}=z_{B}^{0}$. Therefore, $z=z^{0}$. For $z_{N_{f r}} \neq 0$, we have $z=z^{0}+W z_{N_{f r}}$. As $W$ is a basis for the lineality space of $C$, it follows that $z \in \bar{z}^{0}+\operatorname{lin} C$. As $z^{0}$ is an implicit extreme point, $z$ is also an implicit extreme point by Proposition 3.2 in Section 3 .

From the first four equations of the given system of equations, we see that $M z+q \in \operatorname{aff}\left(N_{C}(z)\right)$. 


\section{Worst-case performance comparison: AVI vs MCP reformulation}

In this section, we introduce the MCP reformulation of an AVI and analyze worst-case performance of the two formulations in Sections 5.1 and 5.2 respectively. We assume that the MCP reformulation is solved using the same complementary pivoting method as the AVI formulation is. Computational results comparing the two formulations are presented in Section 6 , and demonstrate the effectiveness of working on the different manifold. (See Tables 13 and Fig. 3 in Section 6 )

\subsection{MCP reformulation}

A linear MCP is defined as follows: for an affine function $F(z)=M z+q$ and a box constraint $B_{1}:=$ $\Pi_{j=1}^{n}\left[l_{j}, u_{j}\right], z$ is a solution to the $\operatorname{MCP}\left(B_{1}, q, M\right)$ if $M z+q=w-v, z \in B_{1}, w, v \in \mathbb{R}_{+}^{n},(z-l)^{T} w=0$, and $(u-z)^{T} v=0$.

It is well known [9, page 4] that an $\operatorname{AVI}(C, q, M)$ can be reformulated as an $\operatorname{MCP}\left(B_{1} \times B_{2}, \tilde{q}, \tilde{M}\right)$, where

$$
\begin{array}{ll}
B_{1}=\Pi_{j=1}^{n}\left[l_{j}, u_{j}\right], & B_{2}=\left\{\lambda \in \mathbb{R}^{m} \mid \lambda \in K^{D}\right\}, \\
\tilde{M} & =\left[\begin{array}{cc}
M & -A^{T} \\
A & 0
\end{array}\right], \quad \tilde{q}=\left[\begin{array}{c}
q \\
-b
\end{array}\right] .
\end{array}
$$

(MCP-reform)

By [14, Proposition 1.2.1], $z^{*}$ is a solution to the $\operatorname{AVI}(C, q, M)$ if and only if there exists $\lambda^{*}$ such that $\left(z^{*}, \lambda^{*}\right)$ is a solution to the $\operatorname{MCP}\left(B_{1} \times B_{2}, \tilde{q}, \tilde{M}\right)$. Therefore we can solve an $\operatorname{AVI}(C, q, M)$ by solving its $\operatorname{MCP}\left(B_{1} \times B_{2}, \tilde{q}, \tilde{M}\right)$ reformulation and vice versa. The solver PATH [8], one of the most efficient MCP solvers, uses this MCP reformulation when it takes an AVI.

Although the two formulations are equivalent, they don't have the same theoretical properties. This is mainly because they look at different feasible regions, which also results in different PL manifolds on which the complementary pivoting is performed. For the $\operatorname{MCP}\left(B_{1} \times B_{2}, \tilde{q}, \tilde{M}\right)$ reformulation, a $\mathrm{PL}(n+m+1)$ manifold $\mathscr{M}_{B_{1} \times B_{2}}$ is built where the full-dimensional cells are defined by the nonempty faces and the normal cones of the set $B_{1} \times B_{2}$, which doesn't consider the polyhedral constraints $A z-b \in K$. For the $\operatorname{AVI}(C, q, M)$ formulation, a PL $(n+1)$-manifold $\mathscr{M}_{C}$ is constructed based on the nonempty faces and normal cones of $C$, which incorporates the polyhedral constraints $A z-b \in K$ explicitly.

\subsection{Worst-case performance analysis}

In worst-case, the complementary pivoting method may end up having traversed all the full-dimensional cells of the underlying PL manifold. As each iteration of the complementary pivoting method corresponds to the traversal of one full-dimensional cell assuming nondegeneracy or lexicographic pivoting, the maximum number of iterations is the total number of the full-dimensional cells, which is finite but could be exponential in the number of constraints. Therefore we compare worst-case performance of the two formulations by counting the number of the full-dimensional cells of the PL manifold each formulation generates.

By construction, the number of the full-dimensional cells is equivalent to the number of the nonempty faces of the polyhedral convex set being considered [24, page 6]. Thus we count the number of the nonempty faces of the sets $B_{1} \times B_{2}$ and $C$ each to compare worst-case performance.

Let $\operatorname{NNF}(S)$ denote the number of the nonempty faces of a polyhedral convex set $S$. To count the number of the nonempty faces, we start with building blocks defining a polyhedral convex set: intervals $[l, u]$ in $\mathbb{R}$ and linear constraints $a^{T} z-b \in K$. For a closed interval $[l, u]$ in $\mathbb{R}$, the number of the nonempty faces is as 
follows.

$$
\operatorname{NNF}([l, u])= \begin{cases}1 & \text { if }-\infty=l<u=\infty \text { or }-\infty<l=u<\infty \\ 2 & \text { if }-\infty=l<u<\infty \text { or }-\infty<l<u=\infty \\ 3 & \text { if }-\infty<l<u<\infty .\end{cases}
$$

For a halfspace or a hyperplane defined by a linear constraint $a^{T} z-b \in K$ where $a \neq 0$ and $b \in \mathbb{R}$, the number of the nonempty faces is as follows:

$$
\operatorname{NNF}\left(\left\{z \in \mathbb{R}^{n} \mid a^{T} z-b \in K\right\}\right)= \begin{cases}2 & \text { if } K=\mathbb{R}_{+} \text {or } K=\mathbb{R}_{-}, \\ 1 & \text { if } K=\{0\}\end{cases}
$$

Based on (8) and (9), we can compute an upper bound on the number of the nonempty faces of a polyhedral convex set.

Lemma 5.1. Let $C$ be a polyhedral convex set defined by $C=\left\{z \in \mathbb{R}^{n} \mid A z-b \in K, l \leq z \leq u\right\}$. Then

$$
N N F(C) \leq \Pi_{j=1}^{n} N N F\left(\left[l_{j}, u_{j}\right]\right) \times \Pi_{i=1}^{m} N N F\left(\left\{z \in \mathbb{R}^{n} \mid A_{i, z}^{T} z-b_{i} \in K_{i}\right\}\right),
$$

where the symbol $\Pi_{j}$ denotes multiplication over indexed terms.

Proof. Let $C_{j}=\left\{z \in \mathbb{R}^{n} \mid z_{j} \in\left[l_{j}, u_{j}\right]\right\}$ for $j=1, \ldots, n$ and $C_{n+i}=\left\{z \in \mathbb{R}^{n} \mid A_{i,:}^{T} z-b_{i} \in K_{i}\right\}$ for $i=1, \ldots, m$. Then $C=\cap_{i=1}^{n+m} C_{i}$. By [25, Corollary 4.2.15], $F$ is a face of $C$ if and only if $F=\cap_{i=1}^{n+m} F_{i}$ where $F_{i}$ is a face of $C_{i}$ for $i=1, \ldots, n+m$. The result follows.

In Lemma 5.1 there could be a large gap between $\mathrm{NNF}(C)$ and its upper bound. The upper bound counts all the possible combinations of the faces of each constraint regardless of their feasibility. When $C$ has only box constraints, i.e., $C=\left\{z \in \mathbb{R}^{n} \mid l \leq z \leq u\right\}$, then equality holds in (10). But, in other cases, the upper bound could be much larger than $\operatorname{NNF}(C)$ as not every combination corresponds to a nonempty face of $C$. For example, if $C=\left\{z \in \mathbb{R}^{2} \mid z_{1}+z_{2} \geq-1,-z_{1}+z_{2} \geq-1, z_{1}-z_{2} \geq-1,-z_{1}-z_{2} \geq-1,-1 \leq z_{1}, z_{2} \leq 1\right\}$, we have $\operatorname{NNF}(C)=9$. However, the upper bound is 144 . It turns out that there are many infeasible combinations, i.e., all the combinations having $z_{1}=-1$ and $z_{2}=1$.

Using Lemma 5.1, we prove that maximum number of the complementary pivoting iterations of the $\operatorname{AVI}(C, q, M)$ formulation is smaller or equal to the one of its $\operatorname{MCP}\left(B_{1} \times B_{2}, \tilde{q}, \tilde{M}\right)$ reformulation.

Proposition 5.1. Let an $A V I(C, q, M)$ formulation and its $M C P\left(B_{1} \times B_{2}, \tilde{q}, \tilde{M}\right)$ reformulation defined in MCP-reform be given. Then the number of the full-dimensional cells of the PL $(n+1)$-manifold $\mathscr{M}_{C}$ is less than or equal to the number of the full-dimensional cells of the PL $(n+m+1)$-manifold $\mathscr{M}_{B_{1} \times B_{2}}$.

Proof. By [25, Proposition 4.2.12], $\mathrm{NNF}\left(B_{1} \times B_{2}\right)=\mathrm{NNF}\left(B_{1}\right) \times \mathrm{NNF}\left(B_{2}\right)$. By applying the same proposition, we have $\operatorname{NNF}\left(B_{1}\right)=\Pi_{j=1}^{n} \operatorname{NNF}\left(\left[l_{j}, u_{j}\right]\right)$ and $\operatorname{NNF}\left(B_{2}\right)=\prod_{i=1}^{m} \operatorname{NNF}\left(\left[l_{i}^{\lambda}, u_{i}^{\lambda}\right]\right)$ where $l_{i}^{\lambda}$ and $u_{i}^{\lambda}$ are lower and upper bounds on $\lambda_{i}$ variable. Using [8] and (9), we see that $\Pi_{i=1}^{m} \operatorname{NNF}\left(\left\{z \in \mathbb{R}^{n} \mid A_{i \bullet}^{T} z-b_{i} \in K_{i}\right\}\right)=$ $\Pi_{i=1}^{m} \mathrm{NNF}\left(\left[l_{i}^{\lambda}, u_{i}^{\lambda}\right]\right)$. By Lemma 5.1, the result follows.

Based on Proposition 5.1, we expect that PATHAVI will have fewer iterations than PATH, which solves the MCP reformulation. See computational results in Sections 6.36 .5 


\section{Computational results}

In this section, we present computational results of PATHAVI highlighting its computational benefits of preserving the problem structure and its robustness and efficiency compared to PATH version 4.7 [8, 16], an established solver for AVIs which uses the MCP reformulation. Section 6.2 compares performance of PATHAVI between the original AVI formulation containing nontrivial lineality space and its equivalent reduced form that does not contain lines. Sections 6.3 6.5 compare performance of PATHAVI and PATH over friction contact problems, compact sets, and Nash equilibrium problems, respectively.

All experiments were performed on a Linux machine with Intel Xeon(R) E7-4850 2.00GHz processor and 256GB of memory. PATHAVI was compiled using GNU gcc version 4.4.7 and its interfaces were linked to GAMS. All problem instances were written in GAMS using the EMP syntax for variational inequalities [15]. We set the time limit to 1 hour and iteration limit to $10^{5}$.

\subsection{Friction contact problem}

Coulomb or dry friction is a ubiquitous phenomenon when mechanical systems interact via contact with each other. Let two bodies be in contact at one point with $u:=\left(u_{n}, u_{t}\right)^{T} \in \mathbb{R}_{+} \times \mathbb{R}^{2}$, the relative (or local) velocity between them. The Coulomb friction phenomenon is described by

$$
\begin{cases}\text { If } u_{t}=0 & \text { then } r \in K_{\mu} \\ \text { If } u_{n}=0 \text { and } u_{t} \neq 0 & \text { then } r \in \text { bdry } K_{\mu} \text { and } \exists \alpha \geq 0 \text { such that } r_{t}=-\alpha u_{t},\end{cases}
$$

where $r:=\left(r_{n}, r_{t}\right)^{T}$ is the contact force. The friction cone $K_{\mu}:=\left\{(t, x) \mid t \in \mathbb{R}_{+}, x \in \mu t D\right\}$, with $\mu>0$ and $D$ the unit disk in $\mathbb{R}^{2}$, defines the admissible set for the contact force. There is a host of approaches to computing a solution to this problem, see [2] for a list of them. In the following, we use a variational approach that can be traced back to at least [17]. We reformulate (11] using normal cone inclusions: $-u_{n} \in$ $N_{\mathbb{R}_{+}}\left(r_{n}\right)$ and $-u_{t} \in N_{r_{n} \mu D}\left(r_{t}\right)$. We cast this problem as a second order LCP (SOLCP): together with the relations $M v=H r+f$ (discretized dynamics) and $u=H^{T} v+w$ (transformation from the global velocity to the local one), we obtain

$$
0 \in\left(\begin{array}{ccc}
M & -H & 0 \\
H^{T} & 0 & E \\
\bar{H}^{T} & 0 & E
\end{array}\right)\left(\begin{array}{c}
v \\
r \\
y
\end{array}\right)+\left(\begin{array}{c}
-f \\
w \\
\bar{w}
\end{array}\right)+N_{X}\left(\begin{array}{l}
v \\
r \\
y
\end{array}\right) .
$$

The matrix $\bar{H}^{T}$ and vector $\bar{w}$ are the same as $H^{T}$ and $w$, except that $\left(\bar{H}^{T}\right)_{i \bullet}=0$ and $\bar{w}_{i}=0$ if $i \bmod 3 \equiv 1$. Similarly, $E \in \mathbb{R}^{3 n_{c} \times 3 n_{c}}$ is defined as $E_{i j}=1$ if $i=j$ and $i \bmod 3 \equiv 1$, otherwise $E_{i j}=0$. The solution of the SOLCP has to lie in $X:=\mathbb{R}^{n_{\text {dof }} \cdot n_{d}} \times K \times K, K:=\Pi_{k=1}^{n_{c}} K^{k}$, with $n_{\text {dof }}$ the number of degree of freedom, $n_{d}$ the number of bodies and $n_{c}$ the number of contacts. The number of degree of freedom depends on the type of system we consider, i.e. if we have rigid bodies, $n_{\text {dof }}=6$. However, if we have deformable bodies, then this number is typically larger and depends on the modeling used. The cone $K$ is not polyhedral. So we need to approximate $K$ to get an AVI from (12). To find a solution to the SOLCP, we would have to solve a sequence of AVIs until one of the solutions also satisfies (12) up to the tolerance. However, we focus here on the case where it makes sense to perform a ray start. Hence, we solve the AVI that would correspond to the first iteration and with an anisotropic approximation of $K$. For each contact we construct a finitely representable approximation $D_{p}^{k}$ of the disk $\mu^{k} D$. Then the cone $K$ is approximated by $K_{p}:=\Pi_{k} K_{p}^{k}$, with $K_{p}^{k}:=\left\{(t, t x) \mid t \in \mathbb{R}_{+}, x \in D_{p}^{k}\right\}$. Finally, with a slight abuse of notation, we redefine $X:=\mathbb{R}^{n_{\mathrm{dof}} \cdot n_{d}} \times K_{p} \times K_{p}$ to refer to (12) as an AVI. It can be verified that PATHAVI processes the AVI (12) if $w \in(\operatorname{ker} H \cap K)^{D}$ by 


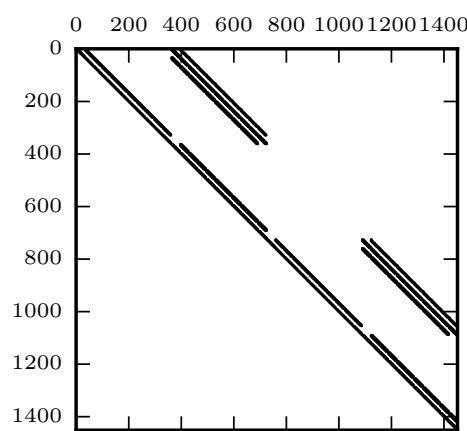

(a) Nonzero patterns of $M$

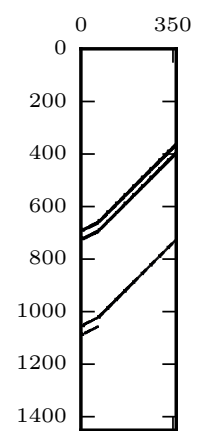

(b) Nonzero patterns of $H$

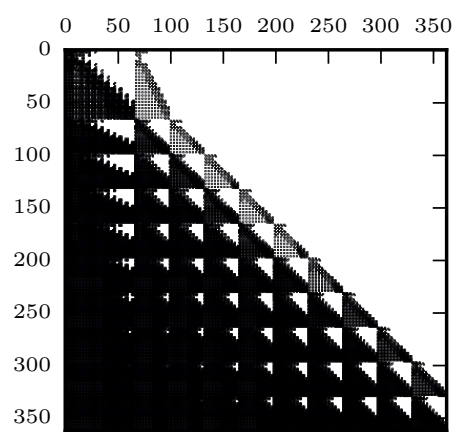

(c) Nonzero patterns of $W$

Figure 1: Nonzero patterns of the matrices $M$ (size: $1452 \times 1452$, nnz: 11330 ), $H$ (size: $1452 \times 363$, nnz: $1747)$ and $W:=H^{T} M^{-1} H$ (size: $363 \times 363$, nnz: 56770$)$.

applying Lemma 3.1 It is noteworthy that this condition is exactly the one given in [18] for the existence of solution to the SOLCP (12). If we solely rely on the $L$-matrix property, we need to assume that $\operatorname{ker} H=\{0\}$, which fails in many instances, for example when a 4-legged chair is in contact with a flat ground.

\subsection{Computational benefits of preserving the problem structure}

The problem data for the following numerical results were obtained from simulations of deformable bodies with the LMGC90 [11] software and using a solver from SiCONOS [3]. In the following, we focus on a simple example where 2 deformable cubes are on top of another. During the simulation, the number of contacts varies between 80 and 120. The shape of $M$ and $H$ is given in Fig. 1. It is noteworthy that if we have to remove the lineality space, that is compute $W$, then all the structure of the problem is destroyed (see Fig. 1c): if we remove the lineality space, the number of nonzero elements increases by a factor of 5 ! It is expected that the linear algebra computations will be more expensive in the reduced space than in the original one because of this large increase of nonzero entries. This has been verified on instances that have the same kind of structure as the matrices depicted in Fig. 1. As shown on Fig. 2. PATHAVI working in the original space is always faster and most of the time is at least twice as fast as PATHAVI working in the reduced space. The time in the reduced space does not take into account the transformation of the problem data (computation of the $W$ matrix).

\subsection{Multibody friction contact problems}

When the bodies are rigid, it is common in the contact mechanic community to eliminate the velocity $v$. The problem is formulated in a reduced space $K_{p} \times K_{p}$ (defined in Section 6.1 and the AVI is

$$
0 \in\left(\begin{array}{ll}
W & E \\
\bar{W} & E
\end{array}\right)\left(\begin{array}{l}
r \\
y
\end{array}\right)+\left(\begin{array}{l}
\omega \\
\bar{\omega}
\end{array}\right)+N_{K_{p} \times K_{p}}\left(\begin{array}{l}
r \\
y
\end{array}\right),
$$

where $W:=H^{T} M^{-1} H$ and $\bar{W}:=\bar{H}^{T} M^{-1} H, \omega:=w+H^{T} M^{-1} f$ and $\bar{\omega}:=\bar{w}+\bar{H}^{T} M^{-1} f$. The lineality space is then trivial in this formulation. 


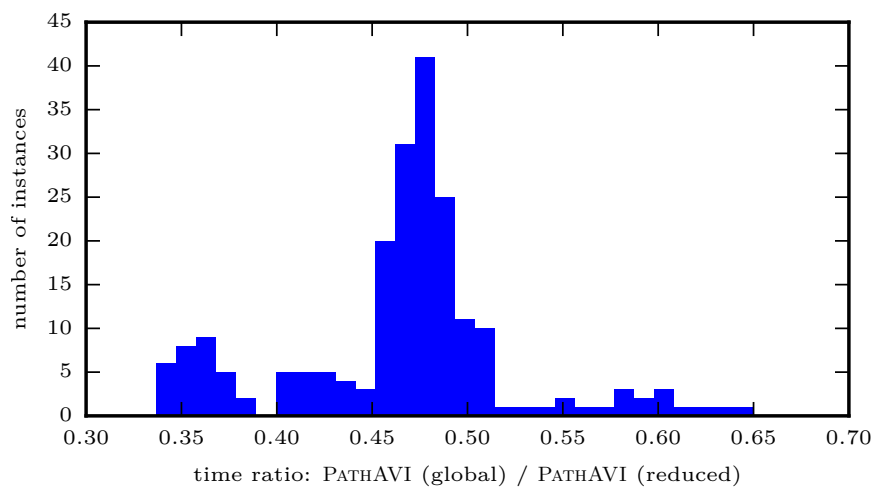

Figure 2: Comparison in terms of speed between the resolution in the original space and the reduced one. The number of iteration was the same for the 209 instances.

We present computational results using the problem data $(W, \mu$ and $q)$ from the FCLIB collection ${ }^{2}[1]$, which aims at providing challenging instances of the friction contact problem. Since we know that PATHAVI can find a solution to any of these examples, the tolerance is set to a low value: $\sqrt{N} \cdot 10^{-9}$, where $N$ is the number of contacts. This value is lower than the default tolerance of PATH (that is already considered quite demanding). First a few observations: PATH fails to perform a ray start on all the examples, due to the fact that $\operatorname{ker} W$ is nontrivial. The results are summarized in Table 1 and show that PATHAVI is more robust than PATH. PATHAVI with the linear algebra package UMFPACK ("pathavi/UMFPACK") solves all

Table 1: Statistics for 4579 friction contact problems of the form (13).

\begin{tabular}{|l|r|r|r|r|r|}
\hline \multirow{2}{*}{ Solver/profile } & \multirow{2}{*}{ \# Failed } & \multicolumn{4}{|c|}{ Failure type } \\
\cline { 3 - 6 } & & Solver error & Stalled & Time & Iteration \\
\hline pathavi/UMFPACK & 0 & 0 & 0 & 0 & 0 \\
pathavi/default & 17 & 0 & 0 & 0 & 17 \\
pathavi/LUSOL-blu & 4 & 0 & 0 & 0 & 4 \\
path/default & 2060 & 535 & 1525 & 0 & 0 \\
path/no crash & 108 & 101 & 0 & 6 & 1 \\
\hline
\end{tabular}

instances, and changing the linear algebra routines to LUSOL ("pathavi/default") leads to a small number of failures. This number can be reduced by using the block-LU updates [13] ("pathavi/LUSOL-blu"). The default behavior of PATH ("path/default") leads to many failures: the crash method is inappropriate for such models. However, even without the crash procedure ("path/no crash"), PATH still fails on more instances than PATHAVI. The different failure types have the following meaning: "Solver error" means that the first basis matrix could not be factorized, despite the use of artificial variables to overcome the rank deficiency. "Stalled" means that a solver possibly tried various strategies but failed to generate solution of the requested accuracy and consequently gave up. Note that this never occurred with PATHAVI on this set of problems. "Time" (or "Iteration") signals that the time (or iteration) limit has been reached. Due to space constraints, we further compare only PATH and PATHAVI with their default settings. First the performance of both solvers in terms of number of iterations is displayed on Fig. 3a. The left plot represents the ratio of the

\footnotetext{
${ }^{2}$ The collection of problem can be freely downloaded by visiting http://fclib.gforge.inria.fr
} 


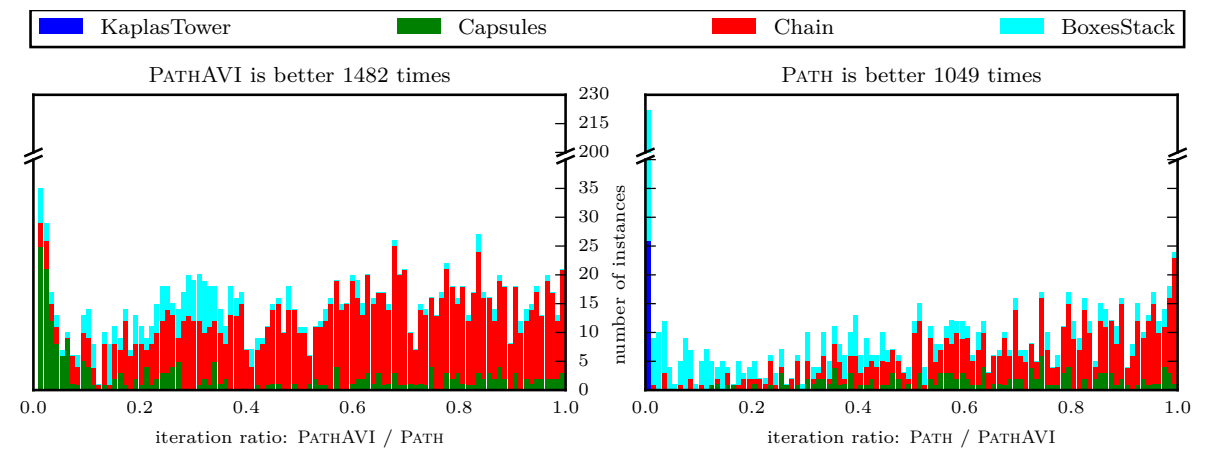

(a) Number of iterations

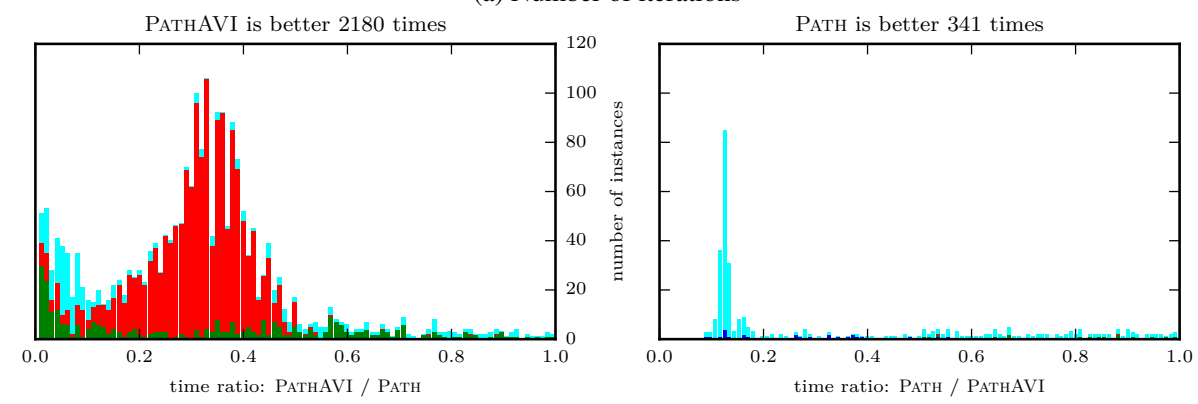

(b) Time

Figure 3: Comparison between PATH and PATHAVI

number of iterations when PATHAVI has a fewer number. For the right plot, it is when PATH solved with a fewer number. Overall, PATHAVI is better than PATH. The spike on the right plot, when PATH finds the solution with a small number of iterations compared to PATHAVI, is explained by the fact that the crash procedure performed well in those cases. However, as it can be seen in Fig. 3b this does not imply that PATH is faster, since those crash iterations can be expensive. If speed is the metric, then PATH is better in less than $10 \%$ of all the instances. The previous spike illustrates that the crash method can find a very good starting point in some instances. This feature of a Newton-based method, which either finds a solution quickly or fails, has already been witnessed when solving friction contact problems. Finally note that when PATHAVI is the faster solver, it usually finds a solution in less than half the time of PATH.

\subsection{AVIs over compact sets}

One strong implication of Theorem 3.1 is that when $C$ is compact (so that rec $C=\{0\}$ ) PATHAVI can process an $\operatorname{AVI}(C, q, M)$ with arbitrary $M$ and $q$. In contrast, this does not hold for the MCP reformulation as the underlying feasible region of it may not be compact although $C$ is compact. This is because whenever the AVI contains polyhedral constraints the associated $\lambda$ variables in the MCP reformulation are unbounded.

We construct 5 AVI instances by taking compact feasible regions from [21] having finite lower and upper bounds and by randomly generating $M$ and $q$ such that the resultant AVI has an $M$ with negative eigenvalues.

Table 2 presents some computational results. As expected, PATHAVI is able to solve all the instances, whereas PATH fails to solve three of them. Also on the two problem instances where both solvers are able to solve, PATHAVI shows 10-30 times fewer iterations, and a similarly decreased elapsed time. These 
Table 2: Performance of PATHAVI and PATH over compact sets

\begin{tabular}{|c|r|r|r|r|r|r|}
\hline \multirow{2}{*}{ Name } & \multirow{2}{*}{ (\#constrs,\#vars) } & \multirow{2}{*}{$(\mathrm{nnz}(\mathrm{A}), \mathrm{nnz}(\mathrm{M}))$} & \multicolumn{2}{|c|}{ Number of iterations } & \multicolumn{2}{|c|}{ Elapsed time (secs) } \\
\cline { 4 - 7 } & & & PATHAVI & PATH & PATHAVI & PATH \\
\hline CVXQP1_M & $(500,1000)$ & $(2495,999)$ & 3119 & fail & 0.459 & fail \\
CVXQP2_M & $(250,1000)$ & $(1746,999)$ & 33835 & fail & 2.827 & fail \\
CVXQP3_M & $(750,1000)$ & $(3244,999)$ & 360 & 3603 & 0.105 & 1.992 \\
CONT-050 & $(2401,2597)$ & $(14597,6407)$ & 11 & 382 & 2.753 & 272.429 \\
CONT-100 & $(9801,10197)$ & $(59197,98875)$ & 3 & fail & 174.267 & fail \\
\hline
\end{tabular}

properties hold for a wide selection of instances and the above table is just provided for expository purposes.

\subsection{Nash equilibrium problems}

Another application of AVIs is to Nash equilibrium problems. In a Nash equilibrium problem, there are multiple agents each of which minimizing its own objective function, and each agent's objective function not only depends on the agent's decision but also other agents' decisions. For example, a typical Nash equilibrium problem computes a solution satisfying

$$
x_{i}^{*} \in \underset{x_{i} \in X_{i}}{\arg \min } h_{i}\left(x_{i}, x_{-i}^{*}\right), \quad \text { for } i=1, \ldots, N .
$$

where we note that each $i$ th agent's objective function $h_{i}$ takes its own decision, denoted by $x_{i}$, and other agents' decisions, denoted by $x_{-i}$.

We generated 6 instances of Nash equilibrium problems, where each $X_{i}$ is a polyhedral convex set and $h_{i}$ is continuously differentiable in $x$ and convex quadratic in $x_{i}$ for each fixed $x_{-i}$. Specifically, $h_{i}$ takes the following form:

$$
h_{i}\left(x_{i}, x_{-i}\right)=\frac{1}{2} x_{i}^{T} Q_{i} x_{i}+x_{i}^{T} Q_{-i} x_{-i}+c_{i}^{T} x_{i}+d_{i}^{T} x_{-i} .
$$

where $Q_{i}$ is symmetric positive definite.

In this case, $x$ is a solution to (NEP) if and only if it is a solution to the $\operatorname{AVI}(C, q, M)$ where $M x+q=$ $\left(\nabla_{x_{i}} h_{i}(x)\right)_{i=1}^{N}$ and $C=\Pi_{i=1}^{N} X_{i}$. The number of agents ranges from 10 to 300 .

Table 3 presents performance of PATHAVI and PATH over the NEPs. The number of iterations of PATHAVI is up to 11 times fewer than PATH. Elapsed time shows similar results except for the last three instances. In those instances, LUSOL has a great difficulty in computing PATHAVI's intermediate basis matrices. If we change the linear algebra engine to UMFPACK, the computation time significantly reduces. Regarding PATH's performance on the last three instances, we would like to point out that the proximal perturbation technique of PATH, which solves a sequence of perturbed MCPs by adding positive diagonal elements $\varepsilon_{k} I$ with $\varepsilon_{k} \rightarrow 0$ as $k \rightarrow \infty$ to the matrix $\tilde{M}$ in (MCP-reform, plays a significant role in its performance. Adding positive diagonals changes the elimination sequence and makes linear algebra computations much faster and more stable. When we turn off the proximal perturbation, PATH either gets much slower than PATHAVI or fails to solve the instance.

\section{Conclusions}

We have presented PATHAVI, a structure-preserving pivotal method for affine variational inequalities. Compared to existing methods, PATHAVI can process an AVI without applying any reduction or transformation 
Table 3: Performance of PATHAVI and PATH over the NEPs

(a) Statistics of the NEPs

\begin{tabular}{|c|r|r|}
\hline Name & (\#constrs,\#vars) & (nnz(A),nnz(M)) \\
\hline vimod1 & $(554,1138)$ & $(4744,22577)$ \\
vimod2 & $(910,1723)$ & $(7935,46137)$ \\
vimod3 & $(1101,2226)$ & $(9117,67634)$ \\
vimod4 & $(870,1828)$ & $(62056,154332)$ \\
vimod5 & $(1327,2586)$ & $(133527,274004)$ \\
vimod6 & $(2210,4359)$ & $(207408,417810)$ \\
\hline
\end{tabular}

(b) \# Iterations and elapsed time of PATHAVI and PATH on the NEPs

\begin{tabular}{|c|r|r|r|r|r|r|}
\hline \multirow{2}{*}{ Name } & \multicolumn{3}{|c|}{ Number of iterations } & \multicolumn{3}{c|}{ Elapsed time (secs) } \\
\cline { 2 - 7 } & PATHAVI & PATH & $\begin{array}{r}\text { PATHAVI/ } \\
\text { UMFPACK }\end{array}$ & PATHAVI & PATH & $\begin{array}{r}\text { PATHAVI/ } \\
\text { UMFPACK }\end{array}$ \\
\hline vimod1 & 367 & 2087 & 367 & 0.372 & 4.129 & 0.437 \\
vimod2 & 319 & 3570 & 319 & 1.098 & 24.134 & 0.645 \\
vimod3 & 590 & 4278 & 590 & 3.208 & 60.553 & 1.639 \\
vimod4 & 1343 & 6146 & 1343 & 127.194 & 66.427 & 18.319 \\
vimod5 & 2167 & 2768 & 2167 & 327.970 & 325.558 & 40.285 \\
vimod6 & 3522 & 4222 & 3522 & 2341.193 & 1841.642 & 109.960 \\
\hline
\end{tabular}

to the problem data even if the underlying feasible region contains lines. PATHAVI can process some newly generated problem classes from applications in friction contact as well as the existing problem class $(L-$ matrices [6]). A computational method for finding a point satisfying sufficient conditions for a ray start is detailed. Through worst-case analysis, we have shown that exploiting polyhedral structure for solving affine variational inequalities is expected to show better performance than using a mixed complementarity problem reformulation. Computational results over friction contact and Nash equilibrium problems demonstrate that PATHAVI compares favorably with PATH in terms of robustness and efficiency.

\section{Acknowledgements}

This work is supported in part by the Air Force Office of Scientific Research and the Department of Energy. The authors are grateful to Steven Dirkse and Todd Munson for comments and suggestions leading to improved computational performance. 


\section{References}

[1] V. ACARy, M. Brémond, T. Koziara, And F. PÉRIgnon, FCLIB: a collection of discrete 3D Frictional Contact problems, Technical Report RT-0444, INRIA, Feb. 2014.

[2] V. ACARY And B. Brogliato, Numerical Methods for Nonsmooth Dynamical Systems: Applications in Mechanics and Electronics, vol. 35 of Lecture Notes in Applied and Computational Mechanics, Springer Berlin Heidelberg, 2008.

[3] V. Acary and F. PÉrignon, An introduction to Siconos, Rapport Technique RT-0340, INRIA, 2007.

[4] R. E. BIXBY, Implementing the simplex method: The initial basis, ORSA Journal on Computing, 4 (1992), pp. 267-284.

[5] M. CAO AND M. C. FERRIS, Lineality removal for copositive-plus normal maps, Communications on Applied Nonlinear Analysis, 2 (1995), pp. 1-10.

[6] _ A pivotal method for affine variational inequalities, Mathematics of Operations Research, 21 (1996), pp. 44-64.

[7] R. Cottle, J.-S. PAng, And R. Stone, The Linear Complementarity Problem, no. 60 in Classics in Applied Mathematics, Society for Industrial Mathematics, 2009.

[8] S. P. DIRKSE AND M. C. FERRIS, The PATH solver: A non-monotone stabilization scheme for mixed complementarity problems, Optimization Methods and Software, 5 (1995), pp. 123-156.

[9] — A pathsearch damped newton method for computing general equilibria, Annals of Operations Research, 68 (1996), pp. 211-232.

[10] C Crash techniques for large-scale complementarity problems, in Complementarity and Variational Problems: State of the Art, SIAM, Philadelphia, PA, 1997.

[11] F. Dubois, M. Jean, M. Renouf, R. Mozul, A. Martin, And M. Bagneris, LMGC90, in 10e colloque national en calcul des structures, Giens, France, May 2011.

[12] B. C. EAVES, A short course in solving equations with PL homotopies, Nonlinear Programming. SIAM-AMS Proceedings, 9 (1976), pp. 73-143.

[13] S. K. Elders Veld AND M. A. SAUNDERS, A block-lu update for large-scale linear programming, SIAM Journal on Matrix Analysis and Applications, 13 (1992), pp. 191-201.

[14] F. FACChinei And J.-S. PAng, Finite-Dimensional Variational Inequalities and Complementarity Problems, vol. I, Springer, New York, 2003.

[15] M. C. Ferris, S. P. Dirkse, J.-H. Jagla, And A. Meeraus, An extended mathematical programming framework, Computers \& Chemical Engineering, 33 (2009), pp. 1973-1982.

[16] M. C. FerRis AND T. S. Munson, PATH 4.7. http://www.gams.com/dd/docs/solvers/path. 2013. 
[17] M. JeAn And J. J. Moreau, Dynamics in the presence of unilateral contacts and dry friction: A numerical approach, in Unilateral Problems in Structural Analysis - 2, G. Del Piero and F. Maceri, eds., International Centre for Mechanical Sciences, Springer Vienna, 1987, pp. 151-196.

[18] A. Klarbring And J.-S. PANG, Existence of solutions to discrete semicoercive frictional contact problems, SIAM Journal on Optimization, 8 (1998), pp. 414-442.

[19] C. E. LEMKE, Bimatrix equilibrium points and mathematical programming, Management Science, 11 (1965), pp. 681-689.

[20] J. LIU, Strong stability in variational inequalities, SIAM Journal on Control and Optimization, 33 (1995), pp. 725-749.

[21] I. MAROS, Qp examples. http://www.doc.ic.ac.uk/ im/OOREADME.QP, 1998.

[22] K. G. MurTy, Linear and combinatorial programming, John Wiley \& Sons, NY, 1976.

[23] S. M. Robinson, Generalized equations and their solutions, part $i$ : Basic theory, Mathematical Programming Study, 10 (1979), pp. 128-141.

[24] - Normal maps induced by linear transformations, Mathematics of Operations Research, 17 (1992), pp. 691-714.

[25] - Convexity in finite-dimensional spaces. Unpublished manuscript, May 2015.

[26] R. T. Rockafellar, Convex Analysis, Princeton University Press, Princeton, NJ, 1970.

\section{Appendix}

Lemma 7.1 (Theorem 4.4 [6]). Consider an AVI $(C, q, M)$ and let $M$ be semimonotone with respect to rec $C$ and invertible on the lineality space of $C$. Suppose that an unbounded ray occurs. Then the value of the auxiliary variable $t$ is constant on that ray and $\Delta z$, the variation in $z$ is nonzero and satisfies

$$
\Delta z \in \operatorname{rec} C, \quad M \Delta z \in(\operatorname{rec} C)^{D}, \quad \text { and } \Delta z^{T} M \Delta z=0 .
$$

Proof. The fact that $\Delta t=0$ and that $\Delta z$ is a solution to (14) follows from the first part of the proof of Theorem 4.4 in [6]. To see that the direction $\Delta \tilde{z}$ is nonzero, we proceed by contradiction: at the current iterate $\left(x^{k}, t^{k}\right)$ we have

$$
G_{\tilde{C}}\left(x^{k}, t^{k}\right)=\tilde{M} z^{k}+\tilde{q}+x^{k}-z^{k}-t^{k} r=0 .
$$

Let $x^{k+1}$ belong to the unbounded ray and suppose that $\Delta z=0$ :

$$
G_{\tilde{C}}\left(x^{k+1}, t^{k}\right)=\tilde{M} z^{k}+\tilde{q}+x^{k+1}-z^{k}-t^{k} r=0 .
$$

It immediately follows that $x^{k+1}=x^{k}$. 
Table 4: Index sets and a basis matrix describing a basic solution $z$ of an LP problem. Assume that $z \in$ $\mathbb{R}^{n}, A \in \mathbb{R}^{m \times n}$, and $b \in \mathbb{R}^{m}$

$B \cup N_{l} \cup N_{u} \cup N_{f r}=\{1, \ldots, n\}$ and $B, N_{l}, N_{u}$, and $N_{f r}$ are mutually exclusive.

$B:=$ a set of basic variables indices

$N_{l}:=$ a set of nonbasic variables indices at their finite lower bounds

$N_{u}:=$ a set of nonbasic variables indices at their finite upper bounds

$N_{f r}:=$ a set of nonbasic free variables indices

$\mathscr{A} \cup \overline{\mathscr{A}}=\{1, \ldots, m\} \quad$ with $\mathscr{A} \cap \overline{\mathscr{A}}=\varnothing$

$\mathscr{A}:=$ a set of active constraints indices, i.e., $A_{\mathscr{A}} \bullet z=b_{\mathscr{A}}$

$\mathscr{A}:=$ a set of inactive constraints indices

$\mathbf{B}=\left[\begin{array}{cc}A_{\mathscr{A} B} & 0 \\ A_{\mathscr{A} B} & \pm I_{\mathscr{A}}\end{array}\right]$ is an invertible basis matrix where $I_{\mathscr{A}}$ is an identity matrix of size $|\overline{\mathscr{A}}| \times|\overline{\mathscr{A}}|$

$z_{B}=A_{\mathscr{A} B}^{-1}\left(b_{\mathscr{A}}-A_{\mathscr{A} N} z_{N}\right), z_{l}=l_{N_{l}}, z_{u}=u_{N_{u}}, z_{N_{f r}}=0, N=N_{l} \cup N_{u} \cup N_{f r}$

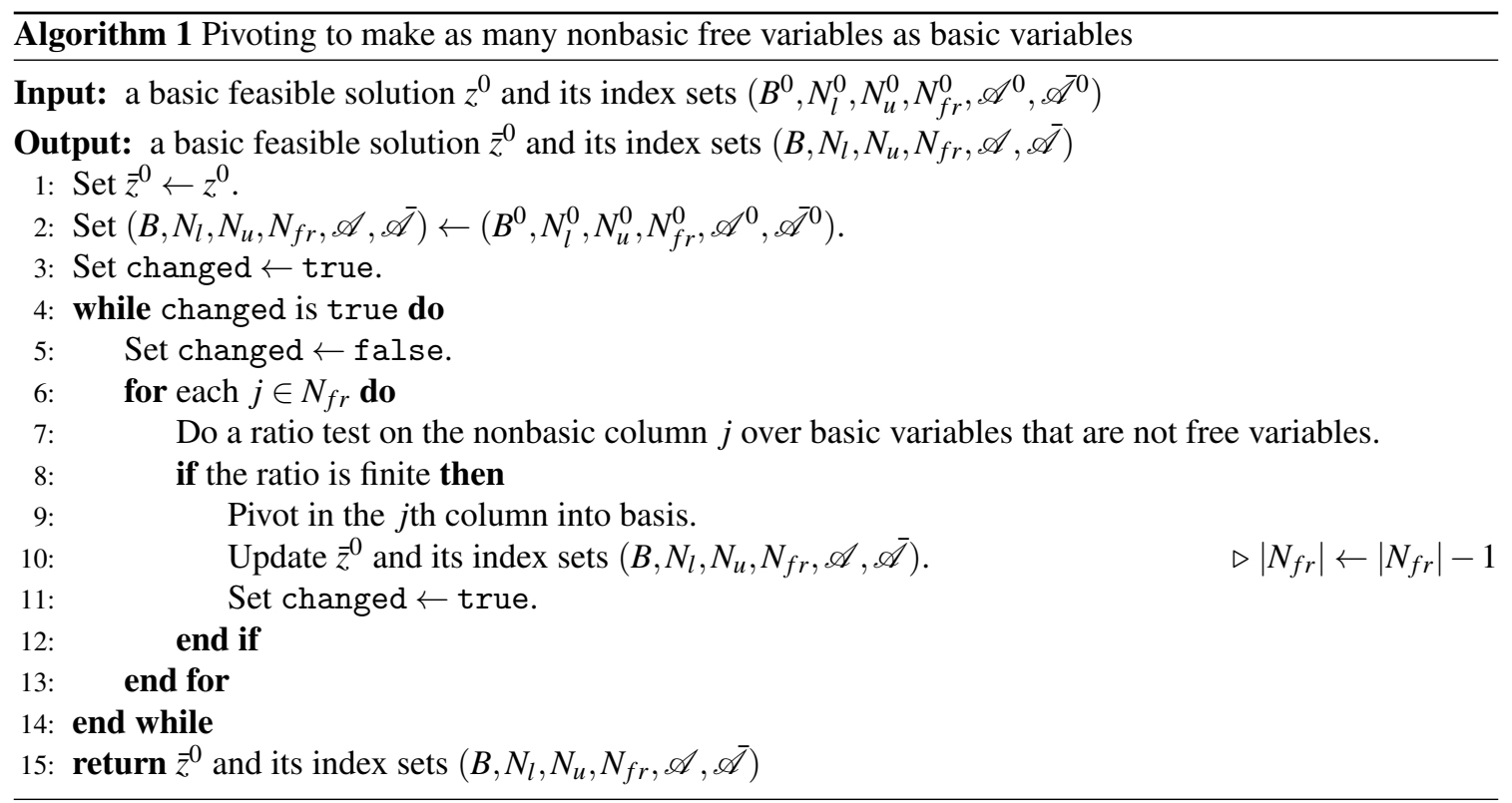

